\title{
Ion Electron-Coupled Functionality in Materials and Devices Based on Conjugated Polymers
}

Magnus Berggren, Xavier Crispin, Simone Fabiano, Magnus J onsson, Daniel Simon, Eleni Stavrinidou, Klas Tybrandt and Igor Zozoulenko

The self-archived postprint version of this journal article is available at Linköping University Institutional Repository (DiVA):

http:/ / urn.kb.se/ resolve?urn=urn:nbn:se:liu:diva-159285

N.B.: When citing this work, cite the original publication.

Berggren, M., Crispin, X., Fabiano, S., J onsson, M., Simon, D., Stavrinidou, E., Tybrandt, K., Zozoulenko, I., (2019), Ion Electron-Coupled Functionality in Materials and Devices Based on Conjugated Polymers, Advanced Materials, 31(22), 1805813.

https:// doi.org/ 10.1002/ adma.201805813

Original publication available at:

https:// doi.org/ 10.1002/ adma.201805813

Copyright: Wiley (12 months)

http:// eu.wiley.com/WileyCDA/ 
[Progress report]

Ion-Electron Coupled Functionality in Materials and Devices based on Conjugated Polymers

Magnus Berggren*, Xavier Crispin, Simone Fabiano, Magnus P. Jonsson, Daniel T. Simon, Eleni Stavrinidou, Klas Tybrandt, Igor Zozoulenko

Laboratory of Organic Electronics, Department of Science and Technology, Linköping University, SE-601 74 Norrköping, Sweden

* Corresponding author: magnus.berggren@liu.se

(Prof. Magnus Berggren, Prof. Xavier Crispin, Asst. Prof. Simone Fabiano, Assoc. Prof. Magnus P. Jonsson, Assoc. Prof. Daniel T. Simon, Asst. Prof. Eleni Stavrinidou, Asst. Prof. Klas Tybrandt, Prof. Igor Zozoulenko)

The coupling between charge accumulation in a conjugated polymer and the ionic charge compensation, provided from an electrolyte, defines the mode of operation in a vast array of different organic electrochemical devices. The most explored mixed organic ion-electron conductor, serving as the active electrode in these devices, is poly(3,4-ethyelenedioxythiophene doped with polystyrelensulfonate (PEDOT:PSS). In this progress report, scientists of the Laboratory of Organic Electronics at Linköping University review some of our achievements derived over the last two decades in the field of organic electrochemical devices, in particular including PEDOT:PSS as the active material. The recently established understanding of the volumetric capacitance and the mixed ion-electron charge transport properties of PEDOT will be described along with examples of various devices and phenomena utilizing this ion-electron coupling, such as the organic electrochemical transistor, ionic-electronic thermodiffusion, electrochromic devices, surface switches and more. One of the pioneers in this exciting research field is Prof. Olle Inganäs and the authors of this progress report wish to celebrate and 
acknowledge all the fantastic achievements and inspiration accomplished by Prof. Inganäs all since 1981.

\section{Introduction}

The coupling between ionic and electronic charge species in conjugated polymers, exemplified by the doping-dedoping switching in the archetypical material of PEDOT (poly(3,4ethyelenedioxythiophene $)^{[1]}$, control the electronic, physical and chemical properties of the material. The dynamic control of this coupling has been utilized in various electrochemical devices combining at least one polymer electrode, an electrolyte and a counter electrode. The dynamic control of this coupling includes accumulation of charges in the polymer and the exchange of ions, provided from the electrolyte. With great inspiration from the early work of Prof. Inganäs, the scientists of Laboratory of Organic Electronics, Linköping University, have explored various concepts of novel electrochemical devices applied into new settings and applications. A selection of our findings, derived over the last 2 decades, are here reported and placed in the context of major achievements done by Prof Inganäs and others within this very exciting research field. Olle, thanks for all inspiration and fun!

\section{Morphology and mixed ion-electron conduction of PEDOT: a theoretical perspective}

Every year more than 1500 papers addressing various aspects of chemistry, structure, physics, and device functionality of PEDOT are reported, which makes this material one of the most studied materials on Earth. ${ }^{[2]}$ At the same time, only a tiny fraction of these publications includes theory and modelling, which represents a strong contrast to many other fields of material science where in silico studies go hand in hand with experimental research. Because of this, many fundamental questions concerning e.g. morphology, nature of the charge carriers, mobility, to name a few, still remain controversial and/or poorly understood. The lack of 
theoretical studies may be related to the complexity of the system at hand, whose description requires a multi-scale modelling encompassing the ångström scale defining the nature of charge carriers, through the nanometer scale determining the morphology of the material, and ending at the micrometer scale featuring the device layout. Only recently, a theoretical understanding of the structural, electrical and ionic properties of PEDOT thin films started to emerge, and in this section, we present a brief account of the current progress focussing on PEDOT as a mixed ion-electron conductor.

\subsection{Morphology of PEDOT.}

Since the pioneering work of Inganäs and collaborators, ${ }^{[3]}$ a number of experimental studies has been reported addressing morphology of PEDOT.(see e.g. ${ }^{[4]}$ and references therein) Theoretical modelling of the morphology of PEDOT doped with molecular tosylate (PEDOT:Tos) using atomistic molecular dynamics simulations was performed by FrancoGonzalez et al. ${ }^{[5]}$ PEDOT:Tos was found to be a largely disordered material, where small crystallites consisting of $3-6 \pi-\pi$ stacked chains (with the stacking distance $3.4 \AA$ ) are embedded in an amorphous matrix of PEDOT chains and are linked by interpenetrating $\pi-\pi$ stacked chains, see Figure 1a to 1f. The crystallization in PEDOT (i.e. formation of the crystallites) occurs already at earlier stages, and the size of crystallites does not change significantly when water evaporates during crystallization. The calculated X-ray diffraction (XRD) pattern (Figure 1g) matches well the corresponding experimental grazing incidence wide-angle X-ray scattering measurements (note that the position of the main peak in the XRD pattern gives the $\pi-\pi$ stacking distance, whereas the width of this peak determines a size of the crystallites according to Scherrer equation $\left.{ }^{[5]}\right)$. During crystallization, the crystallites are assembled in lamellar structure separated by the distance $\approx 12 \AA$, see Figure $\mathbf{1 f}$.

Structural, chemical and dielectric properties of the substrate are known to strongly affect the morphology of deposited polymeric films. Computational microscopy has been carried out to 
reveal the morphology-substrate dependence for PEDOT:Tos thin films deposited on different substrates including graphite, $\mathrm{Si}_{3} \mathrm{~N}_{4}$, silicon, and amorphous $\mathrm{SiO}_{2} \cdot{ }^{[6]}$ It was demonstrated that the substrate strongly promotes a formation of the lamellar structure. It was also shown that the substrate is instrumental in the formation of a specific motif of molecular packing of the polymer on the interface with the substrate (i.e., edge-on or face-on orientations). In particular, Si substrate promotes the face-on orientation because of the strong interaction between PEDOT chains and Si surface. At the same time, $\mathrm{SiO}_{2}$ surface is highly porous and hydrophilic. As a result, a thin layer of water is present on the $\mathrm{SiO} 2$, which reduces the substrate-polymer interaction and promotes the edge-on orientation because of the hydrophobicity of PEDOT (Figure 1 j-k).

\subsubsection{Electron transport in PEDOT.}

A multi-scale modelling of transport properties of PEDOT thin films are recently performed by Rolland et al. ${ }^{[7]}$ relating the electron mobility to the physicochemical properties of the system. The modelling starts from molecular dynamics simulations to generate realistic polymer morphologies, see Figure 2a. Based on the calculated morphology the quantum mechanical calculation of the transfer integrals between states in polymer chains were performed and then used to compute corresponding hopping rates using the Miller-Abrahams formalism, Figure 2b. Then a transport resistive network connecting all the polymeric chains was constructed, Figure 2a and c, and the mobility was calculated using a mean-field approach. Systems with different chain lengths $N=3-18$ and the hydration levels $W=13 \%$ and $43 \%$ w.t. were considered. It was found that the mobility increases exponentially until the chain length reaches $N \approx 15$ and then saturates, see Figure 2f. This behaviour is explained in terms of the percolation thresholds and the transfer integral distribution $P\left(|H|^{2}\right)$. In particular, $P\left(|H|^{2}\right)$ shows a pronounced peak corresponding to the efficient hopping between $\pi$ - $\pi$ stacked chains (peak “A”, Figure $2 \mathbf{d}$ and e), whereas misaligned chains (corresponding to low hopping rates) give rise to a peak "B". As 
the chain length is increased, the percolative threshold moves towards the peak " $\mathrm{A}$ ", leading to the effective transport network connecting all chains in the system through the effective $\pi-\pi$ hopping. Multiscale transport calculations have been also performed to investigate how the character of the substrate affects the electron mobility. ${ }^{[6]}$ It is demonstrated that good crystallinity (PEDOT on graphite substrate) and high content of edge-on (PEDOT on SiO2 substrate) are not enough to achieve the highest electrical in-plane mobility. Instead, the least ordered material with lower degree of the edge-on content (PEDOT on silicon substrate) provides the highest mobility because it exhibits an efficient network of $\pi-\pi$ stacked chain extending throughout the entire sample, see Figure $\mathbf{1 l}$.

\subsubsection{Mixed electronic and ionic conductivity in PEDOT}

A distinguished property of PEDOT is that it supports both electronic and ionic conductivities. It is therefore widely used in variety of devices relying on mixed electron-ion transport including supercapacitors, electrochromic displays, electrochemical transistors, organic biosensors and bioelectronics and many other. The diffusion of $\mathrm{Na}+$ and $\mathrm{Cl}-$ ions in PEDOT:Tos was computationally studied by Modarresi et al. using the coarse-grained molecular dynamics simulations. ${ }^{[8]}$ It was found that the diffusion coefficients decrease exponentially when the hydration level is decreased, see Figure $\mathbf{2 g}$. This behaviour is related to the evolution of the water clusters and to trapping of ions near the polymer matrix as the hydration level is reduced. Although a number of studies have reported modelling of the devices utilizing mixed electronion transport (see e.g. ref. [4] and references therein), a fundamental knowledge and accurate quantitative models of the coupled ion-electron functionality have been to a large extent missing. In particular, the reported models cannot reproduce the capacitive charging and the effect of the intrinsic capacitance without introducing a phenomenological term corresponding to the capacitive current. Recently, a novel model for polymer-electrolyte blends based on the coupling of the chemical potential of the conducting polymer with the electrical double layer 
of the semiconductor-electrolyte interface was introduced by Tybrandt et al.$^{[9]}$ Even though the model is based on the classical drift-diffusion approach, it constitutes a significant departure from previous works as it introduces two distinct material phases with different electrostatic potentials for the electronic and ionic phases. This allows one to introduce an intrinsic capacitance of the PEDOT directly into the Poisson equation, hence overcoming the deficiency of the previous $1 \mathrm{D}$ models where the notion of the intrinsic capacitance was missing. The equations used in this approach are summarized in Figure $\mathbf{2 h}$ for a representative system consisting of metal electrode/PEDOT/electrolyte. The model quantitatively describes a transient and stationary behavior of various experimental devices, see Figure $2 \mathbf{i}$ for the modelling of the organic electrochemical transistor and its comparison to the experimental data.

\section{Organic Electrochemical Transistors}

In organic electrochemical transistors (OECTs), introduced by White et al. for the first time in $1984,{ }^{[10]}$ the electroactive polymer forming the channel is in direct contact with an electrolyte and the source and drain metal electrodes (Figure 3a). Because conjugated polymers can be soft and permeable by design, ions in the electrolyte are able to penetrate into the bulk of the transistor channel under the influence of an electric field. This leads to a modulation of the organic semiconductor redox state (i.e., doping level), and thus to a change of the channel electrical conductivity. The reversible ion exchange and charge compensation process is then at the core of the operational mechanism of OECTs. This makes the OECT an efficient ion-toelectron transducer which is capable of amplifying small chemical signals. A modulation in the gate voltage $\left(V_{G}\right)$ is in fact converted to a modulation in the drain current $\left(I_{D}\right)$ running through the channel. The figure-of-merit that quantifies the efficiency of this transduction is the transconductance, which is defined as $\mathrm{g}=\partial I_{\mathrm{D}} / \partial V_{\mathrm{G}}$. Hence, what makes OECTs different than electrolyte-gated field-effect transistors (EGOFETs) is the coupling between ionic and electronic charges within the entire volume of the channel. In this configuration, individual 
polymer chains, or domains thereof, provide a capacitive interface which leads to a volumetric capacitance being orders of magnitude larger than the electrical double layer capacitance residing at the organic semiconductor/electrolyte interface typical of EGOFETs. The volumetric nature of their response endows OECTs with high transconductance, on the order of $\mathrm{mS}$ for micrometre-scale devices, ${ }^{[11]}$ but also limits their response time to the $\mathrm{kHz}$ frequency range - much lower than the megahertz range achieved by field-effect transistors. ${ }^{[12]} \mathrm{A}$ proper functionalization of the polymer backbone with chemical moieties that promote ion migration throughout the channel ${ }^{[13]}$ as well as the use of redox gate electrodes (e.g., $\mathrm{Ag} / \mathrm{AgCl}$ ) or electrodes with large enough specific surface area (e.g., carbon nanotubes), ${ }^{[14]}$ allows for a fine control of the dimensionality of charge transport (that is, electrostatic field-effect vs bulk electrochemical doping) in these devices.

PEDOT:PSS is a notable example of mixed ion-electron conducting polymer. ${ }^{[15]}$ It represents a class of materials that can yield high transconductance and is one of the most studied p-type conductors, frequently reported in the recent OECT literature. The volumetric doping/dedoping of PEDOT:PSS films results in electrical conductivity changes of several orders of magnitude with consequent modulation of $I_{\mathrm{D}}$. As PEDOT:PSS is oxidized (doped) in its pristine state, and thus highly conducting, the OECTs made thereof operate in the depletion mode. At zero $V_{\mathrm{G}}$, the transistor is in the on state as the sulfonate groups of the PSS compensate the mobile holes in the PEDOT. When a positive $V_{\mathrm{G}}$ is applied, holes in the channel are replaced by cations injected into the active layer and the transistor turns off. In stark contrast, accumulation mode OECTs utilize semiconducting polymers that are undoped in their pristine state as the channel material. In the absence of $V_{\mathrm{G}}$, the device is in the high-resistance off state. When $V_{\mathrm{G}}$ is applied, ions driven into the channel dope the film and increase its conductivity. In this case, mobile carriers are accumulated in the channel and turn the transistor on. Numerous semiconducting conjugated polymers, conjugated polymer composites and conjugated 
polyelectrolytes, transporting both holes (p-type) and electrons (n-type), have been developed and reported as efficient electroactive channel materials for OECTs. ${ }^{[16]}$ A comprehensive description of the latest progress in material design for OECTs can be found in several recent reviews. $^{[17]}$

Because of the very low operating voltages (typically below $1 \mathrm{~V}$ ) and ease of manufacturing, OECTs have attracted a great deal of attention for the development of a variety of applications ranging from printable logic circuits ${ }^{[18]}$ for electronic textiles to drivers for sensors ${ }^{[19]}$ and flat panel display pixels, ${ }^{[20]}$ manufactured on a variety of substrates including soft, low-cost, transparent, and conformable materials (Figure 3b). ${ }^{[21]}$ But, it is with the rise of organic bioelectronics that OECTs are truly experiencing a renaissance. ${ }^{[22]}$ The coupling of ionic and electronic inputs to modulate the transistor channel conductance makes in fact OECTs ideal for interfacing electronics with biological systems. The latter make use of ionic and biochemical currents and gradients for signaling that can be recorded and transduced by OECTs. In addition, because of the soft nature of conjugated polymers, that matches well with the softness of cells and tissues, it is possible to intimately interface conducting polymers with biological systems both at the macroscale, for cell culture analysis, ${ }^{[23]}$ and at the nanoscale, for molecular sensing and cell membrane integration. ${ }^{[24]}$ OECTs have been used for both in vitro and in vivo applications. Because of its high sensitivity and excellent signal-to-noise ratio, OECT can be used for the detection of electrolytes and metabolites, ${ }^{[25]}$ and to monitor the function of electrogenic cells. ${ }^{[26]}$ OECTs can also be used to record electrocardiograms when placed in contact with the human skin (Figure 3c), ${ }^{[27]}$ or to amplify recordings of electrophysiological signals from the brain, heart and muscle (Figure 3d), ${ }^{[28]}$ paving the way toward its use in clinical applications. ${ }^{[29]}$ In addition, OECTs are currently being explored for neuromorphic computing (Figure 3e ${ }^{[30]}$ and artificial afferent nerves (Figure 3f), ${ }^{[31]}$ having recently been shown to exhibit memory functionalities like long-term plasticity and spike-timing dependent 
plasticity, ${ }^{[32]}$ as well as homeostatic plasticity. ${ }^{[33]}$ An attractive feature of OECTs as neuromorphic devices is their potential for low power consumption per switching event, with energy density comparable or even smaller than that of biological synapses. Finally, we recently demonstrated that memory functionality and addressability can be implemented in PEDOT:PSS-based OECTs by coating a ferroelectric polymer layer on the gate electrode. The polarization switching of the ferroelectric polymer, which takes place as the coercive field is overcome, can be used to introduce nonlinearity and bistability in the redox switching of PEDOT:PSS. ${ }^{[34]}$

\section{PEDOT electrochromic devices in color and in the invisible}

Among the most attractive features of PEDOT and other conducting polymers is their ability to undergo redox reactions, which enables applications based on volumetric capacitance and dynamic tuning of their properties after device fabrication. As detailed above, the electrical conductivity varies by orders of magnitude between the oxidized (high conductivity) and reduced (low conductivity) state of the same PEDOT film. In turn, these different versions of the same material possess highly different optical properties. The low-conductivity reduced PEDOT contains neutral segments that contribute to strong absorption of visible light centered around $600 \mathrm{~nm}$, forming an opaque film with a dark blue tint. ${ }^{[35]}$ Charging the polymer by electrochemical oxidation minimizes this absorption, making the same film transparent to visible light. This electrochromic property has made PEDOT popular for low-power reflective display applications and PEDOT electrochromic labels have, for example, been used by Médecins sans Frontières to monitor temperature of medical diagnostic packages delivered to developing countries. Recent advances from our lab also showed possibility to pattern electrochromic PEDOT films by UV light, as well as to make electrochromic freestanding paper. $^{[36]}$ 
Conductive polymer displays are typically monochromic and for PEDOT, the material switches between a dark blue state to a more transparent state. In order to add color functionality, one can combine the electrochromic films with underlying plasmonic metasurfaces, which are ultrathin metallic nanostructured films that, for example, can be designed for high optical reflection in specified wavelength ranges. ${ }^{[37,38]}$ The electrochromic polymer then acts as shutter that controls the reflection from an underlying colorful metasurface. We recently investigated this concept for a plasmonic metasurface based on the abundant metals aluminum and chromium and the system showed highly competitive performance compared with analogues structures based on the more commonly used plasmonic metals gold and silver. (Figure 4a) ${ }^{[38]}$ The reflected color could be tuned between red, green and blue by changing the thickness of a dielectric spacer layer between a top plasmonic chromium nanohole film and a bottom aluminum film mirror. The reflection of the differently colored metasurfaces could then be switched on and off by electrochromic control of a top screen-printed PEDOT:PSS film, also acting as electrode for switching (Figure $\mathbf{4 b}$ and 4c). The concept shows promise for reflective displays in color, with ultra-low power consumption and compatibility with flexible substrates. Looking beyond the visible spectral range reveals that the oxidized form of PEDOT start to show increased absorption when entering the near-infrared and infrared regimes. This is related to the free charge carriers in the conductive material. ${ }^{[35,39]}$ Hence, while the oxidized state provides highest transmission for visible light, the situation is inversed at longer wavelengths, for which the (semi) metallic character of the oxidized film makes it more reflective than the reduced polymer. This suggests that the material's infrared emissivity depends on the redox state of the PEDOT film. We recently investigated the possibility to use this effect to dynamically control the thermal emissivity of thin PEDOT:Tos films, focusing on the wavelength range around $10 \mu \mathrm{m}$ of typical thermal cameras. ${ }^{[40]}$ We found that the thermal emissivity changes significantly when switching between the oxidized and reduced states of the 
PEDOT film. This enabled modulation of the material's apparent temperature as seen by a thermal camera, by almost $10{ }^{\circ} \mathrm{C}$ for a lateral device and by over $6^{\circ} \mathrm{C}$ for a vertical device (Figure 4). Possible applications for this concept include dynamic thermal signage, for example, for use in road signs viewable by thermal safety cameras of modern vehicles. The concept may also be used for active materials with controllable thermal radiation.

\section{Ion-Electron thermodiffusion in conducting polymers}

Heat sources are everywhere. Strategies to harvest electricity from the sun, heat engines, elements and human bodies could lead to new autarkic applications. Thermoelectric phenomena are related to the conversion of heat into electricity by setting a material in a thermal gradient. Conducting polymers displays thermoelectric phenomena, such as the Seebeck effect, which is the creation of a voltage in a material lying in a temperature gradient. Since conducting polymers are electrically conducting, then electrical power can be produced. One of the properties of conducting polymer is that they can transport both ions and electrons. In that context, it is crucial to understand the interplay between electron and ion fluxes in a thermal gradient.

Thermoelectric materials constitute a special class of materials with low thermal conductivity and high electrical conductivity; often described as "a phonon-glass and electron-crystal" material. ${ }^{[41]}$ Three key material properties (electrical conductivity $\sigma$, Seebeck coefficient $\alpha$ and thermal conductivity $\kappa)$ are combined in the so-called dimensionless thermoelectric figure-ofmerit, $\mathrm{ZT}=\left(\sigma \alpha^{2} / \kappa\right) \mathrm{T}$. The importance of this physical quantity lies in its direct relationship with the heat-to-electricity conversion efficiency. ZT quantifies the ratio between the thermal energy passing through the thermoelectric element and the electrical energy produced. The maximum theoretical efficiency is proportional to $(\mathrm{ZT}+1)^{1 / 2}$. A thermoelectric generator consisting with $\mathrm{ZT}=1$ is expected to reach 5\% efficiency if the temperature difference between its hot and cold sides is set to $100 \mathrm{~K}$. 
PEDOT-PSS (Figure 5), is a mixed ion-electron conductor (MIEC). Owning to this property, it has been investigated extensively for its reversible electrochemical activity. ${ }^{[42]}$ High electronic (270S/cm) and ionic conductivity (0.02S/cm @80\%RH) were found for instance in the PEDOT-PSS composite with nanofibrillated cellulose (NFC). ${ }^{[15,43]}$ In the dry state, PEDOT with Tosylate (Tos) or PSS counter ions display attractive thermoelectric properties with the highest reported values for the power factor , $\sigma \alpha^{2}$ of $1270 \mathrm{Wm}^{-1} \mathrm{~K}^{-2},{ }^{[44]}$ and ZT of $0.25,{ }^{[45]}$ $0.31,{ }^{[46]}$ and $0.42,{ }^{[47]}$ at room temperature. In the wet state, PEDOT:PSS is known to transport efficiently both ions and electrons. The combination of electronic and ionic charge carriers leads to complex evolution of the thermo-induced voltage $\left(V_{\text {oc }}\right)$ with time, temperature and/or humidity. Interestingly, the humidity was also found to be a crucial factor, increasing the $\sigma \alpha^{2}$ from 23 to $355 \mu \mathrm{Wm}^{-1} \mathrm{~K}^{-2}$. ${ }^{[48]}$ This rather large increase in $\sigma \alpha^{2}$ was attributed to the morphological changes due to absorption of water and/or electrochemical reactions of PEDOT. A large resulting thermopower, reaching several hundreds of $\mu \mathrm{V} / \mathrm{K}$ at high humidity levels, was identified to be due to primarily ionic Seebeck effects. ${ }^{[49]}$ However, the high $V_{\text {oc }}$ observed in mixed electron-ion conductors, such as for PEDOT-PSS, cannot be sustained, but decreases with time due to the compensation and polarization of ions and electronic charges that occur in such systems.

The set-up used for the $V_{\text {oc }}$ measurement versus time is depicted in Figure 6. Three different polymer layers are characterized, as a temperature difference $(\Delta \mathrm{T})$ of $1{ }^{\circ} \mathrm{C}$ is applied between the two electrodes at $80 \%$ RH. In case of PEDOT:Tos (Figure 5a), the electronic conductor, $V_{\text {oc }}$ increases as $\Delta \mathrm{T}$ increases and saturates at a constant value of $9 \mu \mathrm{V}$ within $\sim 30$ s. Hence, the development of the voltage follows the temperature rise and it is thus almost instantaneous. For PSSNa (Figure 5b), i.e. the ionic conductor, the evolution of $V_{\text {oc }}$ is completely decoupled with the increase characteristics of $\Delta \mathrm{T}$. $V_{\text {oc }}$ reaches a high voltage ranging 5-10 $\mathrm{mV}$ after a period of time of about $100 \mathrm{~min}$; the $\Delta \mathrm{T}$ reaches a static value in typically $30 \mathrm{~s}$. The thermodiffusion of 
ions in polyelectrolytes, due to the Soret effect, is known to lead to large $V_{\text {oc }}$ in the $\mathrm{mV} / \mathrm{K}$ range. ${ }^{[50]}$ For the mixed conductor PEDOT-PSS (Figure 5c), the $V_{\text {oc }}$ evolves in a complex fashion. $V_{\text {oc }}$ increases steadily within $30 \mathrm{~s}$, as $\Delta \mathrm{T}$ is increased, reaching a maximum value (dependent on the $\mathrm{RH}$ ), before the $\Delta \mathrm{T}$ stabilization time. Nonetheless, after that $V_{\text {oc }}$ reaches the maximum value, it then starts to decrease, thus forming a peak. The value then settles to a minimum value of around $4-10 \mu \mathrm{V}$ corresponding to the $V_{\text {oc }}$ of the electronic charge carriers. The peak value of the $V_{\text {oc }}$ depends on the ambient humidity and the value reaches as high as $380 \mu \mathrm{V}$ at $100 \% \mathrm{RH} .{ }^{[51]}$ In comparison to the traditional relatively much smaller electronic contribution (found in PEDOT:Tos), the presence of this high, but short-term lasting, $V_{\text {oc }}$ suggest a dynamic contribution from an ionic Seebeck effect (as found in PSSNa) involved in a complex reorganization of the charge carriers promoting an internal electrochemical reaction within the polymer film. Interestingly, we demonstrate that the time dependence behavior of $V_{\text {oc }}$ is a way to distinguish between three classes of polymeric materials: electronic conductor, ionic conductor and mixed ionic- electronic conductor.

\section{Conducting polymer neural electrodes}

\subsection{The benefit of mixed conductors}

Electrodes can couple the electronic charge carriers in electronics to the ionic charge carriers within biological tissue, thereby acting as a signal transducer between the systems. Current can pass the electrode interface by either charging of an electric double layer (EDL) or through faradaic electrochemical reactions. As reactions can create toxic byproducts, the EDL process is preferable for neural electrodes. ${ }^{[52]} \mathrm{A}$ challenge with EDL-based electrodes is that the amount of charge stored in the EDL of a planar electrode is rather small. Mixed conductors based on conducting polymers (CPs) have provided a solution to this problem, as the dual conduction allows the EDL to form within the bulk of the material, ${ }^{[53]}$ thereby improving the areal capacitance in comparison to planar electrodes by several orders of magnitude. 


\subsubsection{Conducting polymer electrode materials}

The Martin group pioneered the use of CPs as neural electrode coatings in the early 2000s. ${ }^{[54,}$ ${ }^{55]}$ The coatings were fabricated by electropolymerization, a method in which the CP coating is grown through polymerization of monomers from solution by the application of electric current. Electropolymerization has been a very popular method for the fabrication of neural electrodes, as it allows for direct patterning of the electrodes, as well as control of film thickness and morphology ${ }^{[56]}$ By varying the dopant ions and applied voltage/current during polymerization, [57, 58] a wide range of film morphologies can be achieved, which heavily affect the mixed transport within the material. The ionic conduction, which often constitutes the performance limiting factor for CP electrodes, can be effectively tuned by the variation of counter ions and polymerization conditions. ${ }^{[58,59]}$ In most of the early work on CP electrodes polypyrrole (PPy) was used, ${ }^{[54,55,60]}$ which has the advantage that the Py monomer is highly soluble in water. In more recent work, the CP poly(3,4-ethylenedioxythiophene) (PEDOT) has often been the material of choice due to its superior stability. ${ }^{[56,58,61]}$ Derivatives of PEDOT has also been evaluated, ${ }^{[62]}$ although the regular PEDOT remains the dominating material to date. An advantageous property of CPs is that various biomolecules/drugs can be inserted into the electrodes during fabrication. ${ }^{[55,63]}$ The Inganäs group contributed to this topic and showed improved biocompatibility by the inclusion of different biomolecules. ${ }^{[58,64]}$ A common issue for CP electrodes is the stability over time. ${ }^{[65]}$ The loss of functionality have two origins; degradation of the CP or loss of adhesion to the electrode contact. Electrode properties can be improved by growing $\mathrm{CP}$ around a carbon nanotube (CNT) mesh, as the CNTs provide mechanical strength, electrical transport and a porous morphology. ${ }^{[66]}$ Therefore, some of the best electrode performances have been reported for CP-CNT composite electrodes. ${ }^{[67]}$ The stability of CP electrodes can also be greatly improved by the use of iridium oxide or 
nanostructured platinum as electrode contacts, ${ }^{[68]}$ or by electrografting an adhesion layer before CP deposition. ${ }^{[69]}$

The use of chemically polymerized PEDOT:PSS dispersions for flexible electrodes has been pioneered by the Malliaras group. ${ }^{[70]}$ Here the polymer is deposited from solution, which opens up for new fabrication schemes and film morphologies. An advantage of this approach is that arbitrary numbers of electrodes can be patterned in one step, as well as more advanced devices like electrochemical transistors. ${ }^{[71]}$

\subsubsection{Conducting polymer neural interfaces}

Interfacing of the nervous system can provide treatments and diagnosis for a range of neurological diseases and disorders. Today electrical stimulation can treat Parkinson’s disease, epilepsy, deafness, chronic pain and blindness to mention a few. ${ }^{[72]}$ Electrical recording can be used for diagnosis of epilepsy and brain damage, as well as for controlling artificial limbs. CPs can improve the electrode-tissue interface, both with respect to electrical performance as well as biocompatibility. ${ }^{[73]}$ The high areal capacitance of CP electrodes lowers the impedance and provide better signal-to-noise ratio for recordings. This is especially useful for microelectrodes, where sufficient capacitance is needed despite the small electrode area. The benefits of CP electrodes have been demonstrated with neural depth probes, where the CP coatings improved signal quality drastically. ${ }^{[74]}$ The release kinetics of loaded drugs can be tailored by the morphology, e.g. by loading CP nanotubes with anti-inflammatory drugs. ${ }^{[75]}$ In a drastic attempt to bridge the scar tissue surrounding implanted electrodes, PEDOT has even been electropolymerized in vivo. ${ }^{[76]}$

A current trend is to develop ultra-flexible micro-electrode arrays with CP electrodes. ${ }^{[70]}$ These devices are typically made by microfabrication processes where thin films of PEDOT:PSS from dispersion are patterned. These devices are conformal and provide high electrode densities in combination with excellent recording quality, which has allowed scientists to track individual 
neurons from the surface of the brain. ${ }^{[77]}$ The technology has now matured to the degree where the first human trials have been performed. ${ }^{[78]}$ Although flexibility is highly advantageous, it would be even better if the devices were soft and deformable. An attractive route forward would be to apply recent advances in stretchable CPs to inorganic soft and stretchable electrode grids (Figure 7). ${ }^{[79]}$

\section{Iontronics}

Another useful application of ion-electron coupled functionality is in controlled delivery via ion transport. For several decades, researchers have utilized redox processes in conjugated polymers to liberate dopant ions or other embedded substances for drug delivery applications $^{[80]}$. More recently, polyelectrolyte systems such as PSS have been used to control the flow and delivery of these liberated ions with significantly more precision. PSS is a canonical example of a polyanion, mostly known in the organic electronics community as a dopant material for, e.g., PEDOT. PSS is a hygroscopic material and PEDOT:PSS is often utilized in a hydrated state, for example in bioelectronics applications. ${ }^{[22,81]}$ The resulting hydrated PEDOT:PSS thus becomes an efficient cation transporting material. By the process of Donnan exclusion $^{[82]}$, anions are excluded by the fixed negative charge on the PSS, but cations can migrate and diffuse through the bulk of negative charge. In the language of ion transport, hydrated PSS is a cation exchange membrane (CEM). Corresponding hydrated polycations, such as poly(vinylbenzyl chloride) quaternized by dimethylbenzylamine (qPVBC) or poly(vinylbenzyl chloride) quaternized by triphenylphospine (PVBPPh3), can act as anion exchange membranes (AEMs). ${ }^{[33,84]}$

Since AEMs and CEMs are ion transport materials exhibiting majority charge carriers of a given sign, they are directly analogous to n- and p-type materials. Thus, appropriate geometrical combinations of AEMs and CEMs provide a similar range of functionality and device 
architecture those provided by organic and inorganic n- and p-type materials. This is the material and device repertoire behind the recently developed field of “iontronics”, where AEMs and CEMs are used to design specific device functionality and conjugated polymers are often used as the electronic-to-ionic transducer - with a 1-to-1 equivalence between electronic charge passed through the circuit and ions "pumped".

As with conventional electronics, the simplest - and first demonstrated - iontronic component was a resistor, known as the organic electronic ion pump (OEIP). ${ }^{[85,86]}$ The first OEIPs utilized PEDOT:PSS electrodes to convert electronic signals into cation fluxes, which were electrophoretically “pumped” laterally through PSS thin films (Figure 8a and b). These basic OEIPs have been used over the years for a variety of in vitro cell signaling studies ${ }^{[85,87]}$, in vivo studies $^{[86]}$ and therapeutic demonstration ${ }^{[88]}$, and even to modulate plant physiology ${ }^{[89]}$. As with conventional semiconductors, the next phase of this AEM/CEM-based iontronics was to demonstrate diode functionality at AEM-CEM junctions. Iontronic diodes were achieved at the junction of hydrated PSS and qPVBC in so-called ion bipolar membrane diodes (IBMDs, Figure 8c and d). ${ }^{[83]}$ This and subsequent demonstrations included a variety of architectures and materials to enhance on-off ratios and avoid water-splitting ${ }^{[83,84]}$, a common concern in bipolar membrane applications. The most complicated component in the iontronics toolbox has been the ion bipolar junction transistor (IBJT). ${ }^{[90]}$ The IBJT is the analogy of the npn (or pnp) bipolar junction transistor: an AEM input (emitter/source) and output (collector/drain) meet a CEM control electrode (base/gate) at neutral junction (e.g., hydrated polyethylene glycol, PEG) (Figure 8e, the pnp analog is shown in Figure 8f).

In addition to the drug and substance delivery applications described above for OEIPs, complex iontronic circuits utilizing OEIPs, IBMDs, and IBJTs have been demonstrated for more 
advanced applications. IBMDs and IBJTs have been used to create iontronic logic circuits for complex and multiplexed electronic-to-ionic signal transduction and substance delivery. ${ }^{[83,}$ 91] IBMDs and OEIPs have been combined in rectifying circuits which promise to overcome the redox capacity inherent in the PEDOT:PSS driving electrodes. ${ }^{[92]}$ And IBMDs and OEIPs have been combined into an array of individually addressable delivery pixels with significantly faster delivery dynamics than previously achievable. ${ }^{[93]}$ More recently, OEIPs have been combined with microfluidics to enable deep-brain implant form factors with remarkably efficient dynamics and low-voltage operation. ${ }^{[94]}$

Iontronics has thus developed significantly in recent years, with a predominant focus on bioelectronics applications. Within the field of bioelectronics, these first demonstrations of therapies and tools for augmenting cell signalling promise a range of medical technologies that can provide immeasurable help for treatment and wellness. Beyond the field of bioelectronics, iontronics provide a uniquely precise substance delivery system, as well as an entry point into an entirely ion-based world of circuits and systems.

\section{Surface switch devices}

The coupling between ion exchange and charge accumulation in conducing polymers impacts the physical and chemical properties of the bulk of the conjugated system, and it does so also for the outermost surface. This opens up for surface switch devices with an active surface that can be controlled upon electric addressing. In an electrochemical device, typically, the conjugated polymer is in contact with an electrode, which provides electronic charge transfer, and an electrolyte that provides ions. If the polymer serves as its own electrode and if it is placed on top of a solidified electrolyte and a second electrode, electrochemical control of the outermost surface, exposed to air and the environment, is achieved ${ }^{[95]}$ (Figure 9a). Such devices can then be used to control the wettability of for instance water. In one setting, polyaniline 
(PANI), doped with dodecylbenzenesulfonic acid (DBSA), was used as the active material and we found that the lowest water contact angle (highest surface energy) was found for the reduced state (Figure 9b and c). This is somewhat counterintuitive but is explained by that DBSA is more liberated (thanks to the reduction of PANI) and is then prone to re-orientate as water is applied to the surface. So, when water hits the PANI surface, DBSA re-configures and expresses its polar side towards the water, thus producing a high-energetic surface. When Poly(3-hexylthiophene-2,5-diyl) is used as the active material, the oxidized surface expresses a relatively lower water contact angle as compared to the reduced side. In this case, the dipole properties of the bare surface dictate the surface energy properties ${ }^{[96]}$.

Active control over the surface properties is of great importance for cell growth and regenerative medicine applications. It is well known that the energy and charge of the surface impact the ability for seeded cells to adhere, proliferate and grow into tissues. Already in 1994, Ingber and Langer published a report describing how polypyrrole (PPy) was used as to control the growth and spreading of fibroblasts ${ }^{[97]}$. Inspired by these early experiments, we explored PEDOT, doped with tosylate, as the electronic surface to regulate the adhesion and growth of MDCK epithelia cells (Figure 9d and e) ${ }^{[98]}$. We found that the MDCK cells prefer to adhere to the reduced PEDOT surface, expressing well developed nuclear morphology along with other "healthy” cell components (Figure 9f and g). Conversely, on the oxidized side, the MDCK cells adhere to a much less density, then following by apoptosis. As the development of PEDOT-based OECTs developed, we also designed transistor channels onto which MDCK cells were adhered and evaluated. Depending on the transistor bias conditions, we found that MDCK cell density gradients could be generated, which matches the potential (and chargeaccumulation) characteristics of the channel. The origin to control the MDCK in-binding and growth along the PEDOT surface is related to electronic control over the characteristics of matrix proteins. As the PEDOT surface is switched, it controls orientation and also structure of 
matrix proteins, in particular that of fibronectin. Along the reduced PEDOT surface, the RGD receptor of fibronectin is expressed to the incoming MDCK cells, especially this is of great relevance for the transmembrane integrin and heterodimer components (Figure 9e). This coupling favors cell adhesion and growth. In contrast, along the oxidized surface these RGD receptors seems to be hidden, thus suppressing cell adhesion.

In regenerative medicine, not only adhesion, proliferation and spreading are of great interest, also release is important in order to harvest healthy tissues and cell systems for further studies or regeneration. Traditionally, enzymatic ${ }^{[99]}$ approaches are used, such as Trypsin, to impact the cell binding proteins, selectively. This breaks the binding between the cells and the carrying substrate. However, these approaches typically induce severe damage to membrane and extracellular matrix protein of the cells. With electronic surface switches a change in chemical properties can also result in disintegration of the film that can be utilized as a release technology for cells and tissues. With PEDOT-S:H (Figure 9h) coated onto electrodes ${ }^{[100]}$ electronic control over detachment is achieved. As the PEDOT-S:H is oxidized it swells and forms a film including several micro-cracks, which finally detaches from the underlying substrate electrode (Figure 9i). This technique has been evaluated using human epithelial T24 cells and we found an improvement with respect to cell surface antigens as compared to traditional trypsinization.

\section{Electronic Plants}

Organic electronic materials have been explored as active layers in a variety of bioelectronics devices due to their ability to conduct electronic and ionic carriers in a tightly coupled fashion (Figure 10). Therefore, these materials can act as ions to electrons converters offering a handle for communication with the biological world. Bioelectronics devices should not only have high performance but also minimum invasiveness offering mechanical matching with the tissue, biocompatibility and bio-stability. A more sophisticated approach will be direct functionalization of tissue with active materials that are driven by self-organization, self- 
assembly and by specific response to biochemical cues. In this way the biological environment can guide the organization of devices.

One can find in the literature quite a few examples on self-assembly of organic electronic materials to biological constructs such as $\mathrm{DNA}^{[101]}$, proteins ${ }^{[102]}$, cellulose ${ }^{[15]}$, and lipid membranes $^{[103]}$. This approach has two main targets: (i) induce non-native functionality to the biological unit for example electrical conductivity ${ }^{[101,102]}$, specific organization ${ }^{[104]}$, therapy ${ }^{[105]}$, fluorescence tagging ${ }^{[106]}$; and (ii) utilize the biological unit as part of a device for example as structural template $^{[107]}$. Recently we advanced this concept by direct functionalization of plants. Instead of using isolated biological units we used a plant - an advanced organism and demonstrated self-assembly ${ }^{[108]}$ and in vivo polymerization ${ }^{[109]}$ of organic electronic materials introducing the concept of Electronic Plants (e-Plants).

Electronic interface with plants can have many applications from plant control/optimization, to energy and bio-hybrid systems. Using the common rose we demonstrated in vivo fabrication of organic electronic devices where the plant's structure and chemical environment are an integral part of the devices and circuits ${ }^{[108]}$. Initially we used PEDOT-S a self doped PEDOT that self organized and formed conductors within the xylem, the water transporting vascular tissue of the plant. PEDOT-S maintains its electrical and electrochemical properties within the plant tissue allowing us to form more complex devices and circuits. As an example we demonstrated operation of an organic electrochemical transistor and the digital gate NOR.

Although PEDOT-S can be uptaken from the plant it localizes only at the stem. To overcome this limitation we designed a thiophene-based oligomer that can polymerize in vivo ${ }^{[109]}$. ETE-S due to its small molecular size can be distributed in every part of the vascular tissue of the rose cutting, from stem to flower and even cross through the veins and reach the apoplast of leaves. At the same time, it polymerizes in vivo without any external stimuli but with the plant acting as a template and a catalyst. Although the exact mechanism is not well understood we 
hypothesize that the defense mechanism of the plant is triggered, generating reactive oxygen species that accelerate the polymerization. In addition, there is a preferable interaction between the ETE-S and the hydrophobic xylem surfaces that allow condensation of the molecule at the surface. Molecular dynamics reveal that the oligomers form crystallites due to $\pi-\pi$ stacking with percolating paths for the charge carriers ${ }^{[110]}$. This leads to the formation of long conducting wires with $10 \mathrm{~S} / \mathrm{cm}$ conductivity and 20F/g specific capacitance. The long conductors templated by the xylem along with the natural architecture of the plant and the electrolyte rich environment can be used for the fabrication of a supercapacitor along the stem. The supercapcitor exhibits very good stability upon switching and excellent charge retention, consisting the first demonstration of energy storage within a plant.

Apart from the vascular tissue of plants we functionalized the leaves of the rose and fabricated electrochromic pixels ${ }^{[108]}$. Through the leaf pores, the stomata, we infiltrated PEDOT:PSS mixed with nanofibrillated cellulose, NFC, reaching the internal structure of the leaf. PEDOT:PSS-NFC forms free standing and self-organized 2D electrodes that are compartmentalized from the vasculature of the plant. The pixels change color upon application of voltage through external contacts exhibiting homogenous electrochromism in the compartments in direct contact with the external electrodes and electrochromic gradients on the intermediate ones. The type of electrochmism gives information on the transport suggesting mixed conduction within the compartments and pure ionic transport across the veins of the leaves.

E-Plants technology is still at its infancy, but many exciting possibilities arise from the direct functionalization of plants and construction of bio-hybrid systems. As such by establishing coupling between materials and devices with biological organisms, and particularly photosynthetic organisms will open possibilities for both fundamental and applied research and can result in an extended green technology. Bio-hybrid systems can revolutionize our approach 
to technology, with natural processes and systems being an integral part of the technology. Fundamental understanding of the mechanism for self-assembly/organization of materials and in vivo chemistry can lead to rational design of materials and systems and consequently advanced technology.

\section{Acknowledgement}

Financial support for the work at the Laboratory of Organic Electronics at Linköping reported above has been provided from the Knut and Alice Wallenberg Foundation, Vinnova, the Swedish Research Council, the Önnesjö Foundation, the Swedish Foundation for Strategic Research, the European Commission, and the strategic research area of Advanced Functional Materials at Linköping University. 

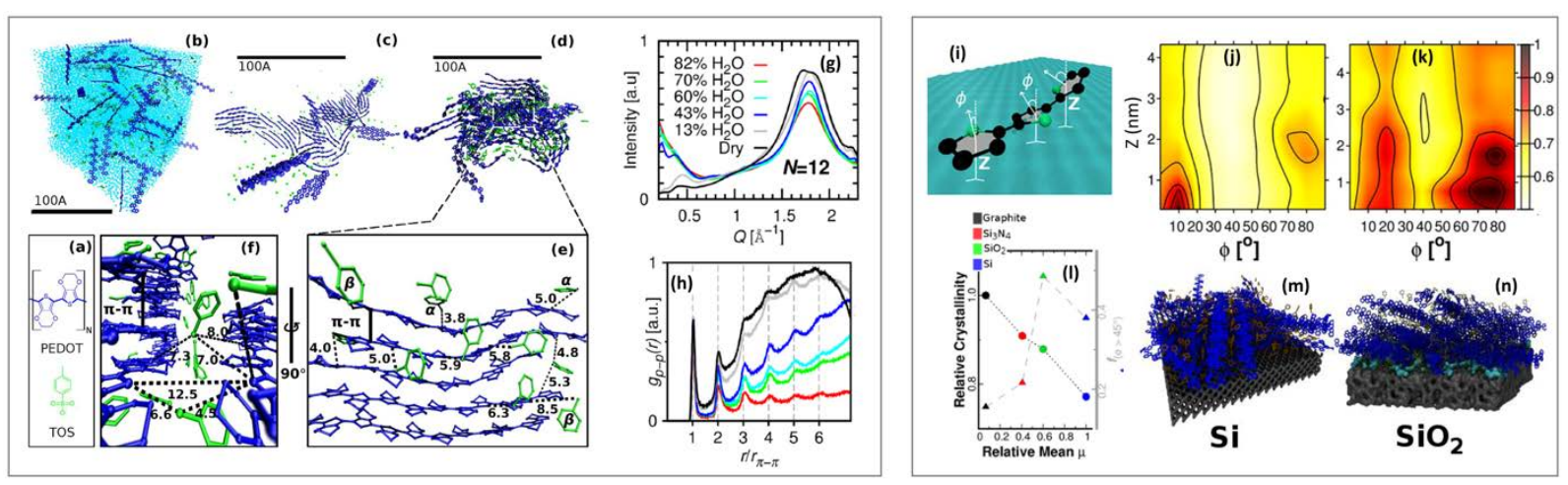

Figure 1. Morphology of PEDOT. (a) Molecular structure of PEDOT and Tos. (b-d) Snapshots of the PEDOT:Tos structures as water is evaporated from the initial random configuration to the dry phase. PEDOT is shown in blue, Tos in green, and water in light blue; for clarity, water is not shown in parts c-f. (b,e) Zoom of a representative crystallite. (f) Zoom of a part of a structure illustrating a formation of the lamellar structure. (g) Calculated XRD pattern for different hydration levels. (h) Radial distribution function $\mathrm{g}_{\mathrm{P}-\mathrm{P}}(\mathrm{r})$ for the distance between PEDOT chains. The number of peaks in $\mathrm{g}_{\mathrm{P}-\mathrm{P}}(\mathrm{r})$ determines the number of PEDOT chains in the crystallite. (j,k) Probability distribution, $\mathrm{P}(\phi, \mathrm{Z})$, illustrating formation of respectively face-on and edge-on structures for $\mathrm{Si}$ and $\mathrm{SiO}_{2}$ surfaces. $\mathrm{Z}$ is the distance of from the surface, a definition of the normal angle $\phi$ is given in (i). (l) Mean mobility vs relative crystallinity and the content of the edge-on configurations, $\mathrm{P}\left(\phi>45^{\circ}\right)$; the mean mobility for different surfaces are normalized to the one of Si. (m,n) Snapshots of PEDOT on Si and SiO2 surfaces (water molecules at $\mathrm{SiO}_{2}$ surfaces are shown in cyan). Parts a-h adapted and reproduced with permission from the American Chemical Society, ref. [5] copyright 2017; parts i-n reproduced with permission the American Chemical Society, ref. [6] copyright 2018. 

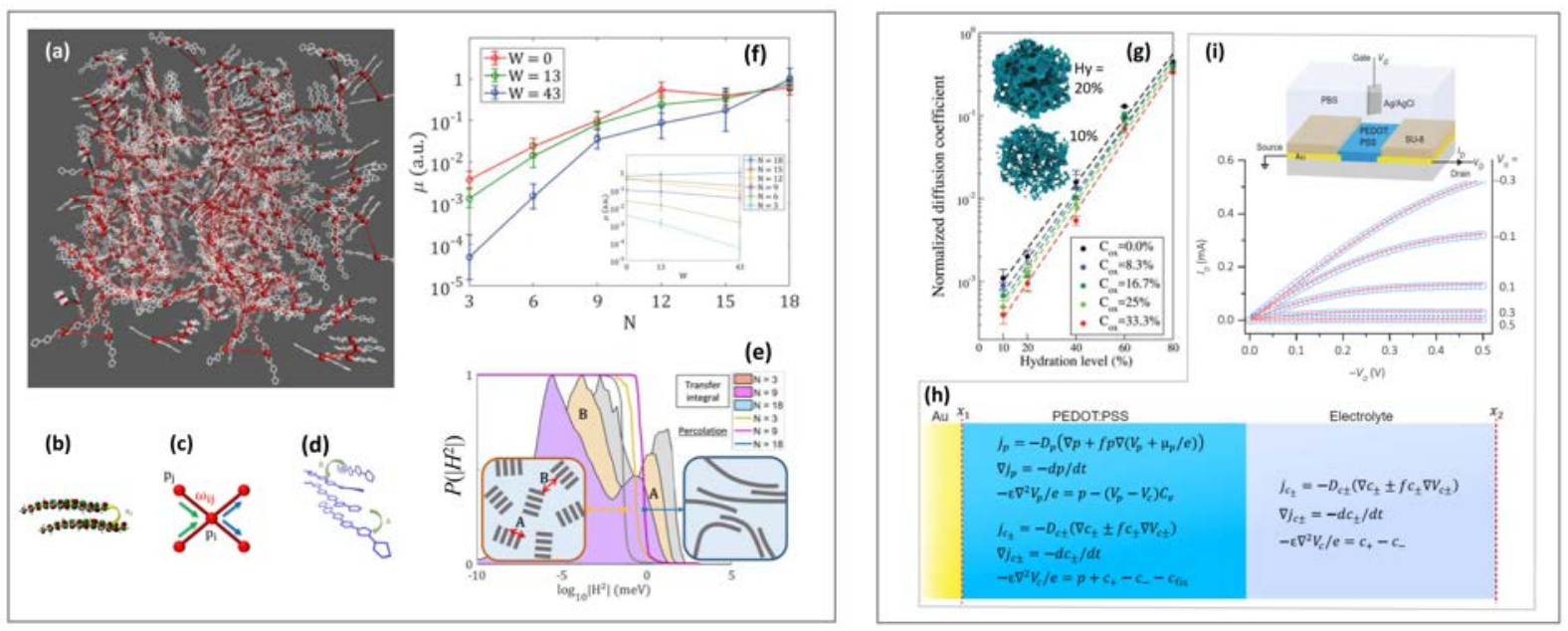

Figure 2. Electronic and ionic transport in PEDOT. (a) A schematic illustration of the calculated PEDOT morphology and corresponding resistive network. (b) Calculation of the overlap integrals $H$. (c) A node $i$ of the resistive network with the occupation probability $p_{i}$, and hopping probabilities $\omega_{\mathrm{ij}}$. (d) Illustration of peaks "A" and "B" in the hopping integral probability distribution $P\left(\left|H^{2}\right|\right)$ shown in (e). (f) Dependence of the mobility on the chain length $\mathrm{N}$ and the water content W (in the inset). (e) Analysis of the mobility in terms of the percolation thresholds and the transfer integral distribution $P\left(|H|^{2}\right)$. (g) calculated ionic diffusion coefficient as a function of the hydration level Hy: insets: water clusters at $\mathrm{Hy}=20 \%, 10 \%$. (i) The measured output characteristics $(\circ)$ of the OECT (shown in the inset) are accurately reproduced (lines) with modelling. (h) Equations used in the modelling of a system consisting of metal electrode/PEDOT/electrolyte. Parts a-e adapted and reproduced with permission from the American Physical Society, ref. [7] copyright 2018; part g adapted and reproduced from ref. [8]; parts i-h adapted and reproduced from ref. [9]. 

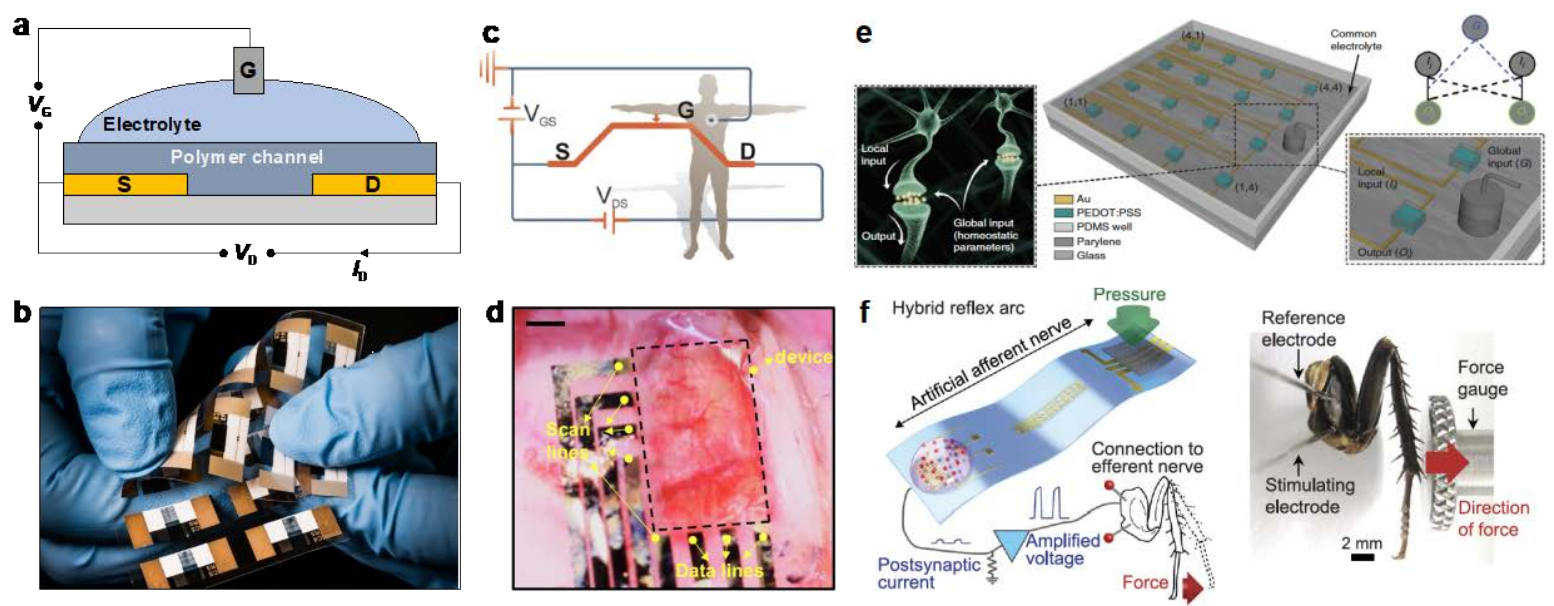

Figure 3. (a) Cross-sectional schematic of the OECT. (b) PEDOT:PSS-based OECTs screen printed on a flexible, transparent substrates. Photo: Thor Balkhed, Linköping University. (c) ECG recording with a bioresorbable OECT operated in direct contact with the skin. Reproduced with permission from John Wiley and Sons, ref. [27] copyright 2014. (d) Transparent electrophysiology arrays on the cortical surface of the optogenetic rat. Reproduced with permission from ref. [111]. (e) Global gate induced effects on an organic neuromorphic device array. Reproduced with permission from ref. [33], copyright 2017 the authors. (f) Artificial flexible afferent nerve connected to biological nerves in a cockroach. Reproduced with permission by AAAS, ref. [112] copyright 2018. 

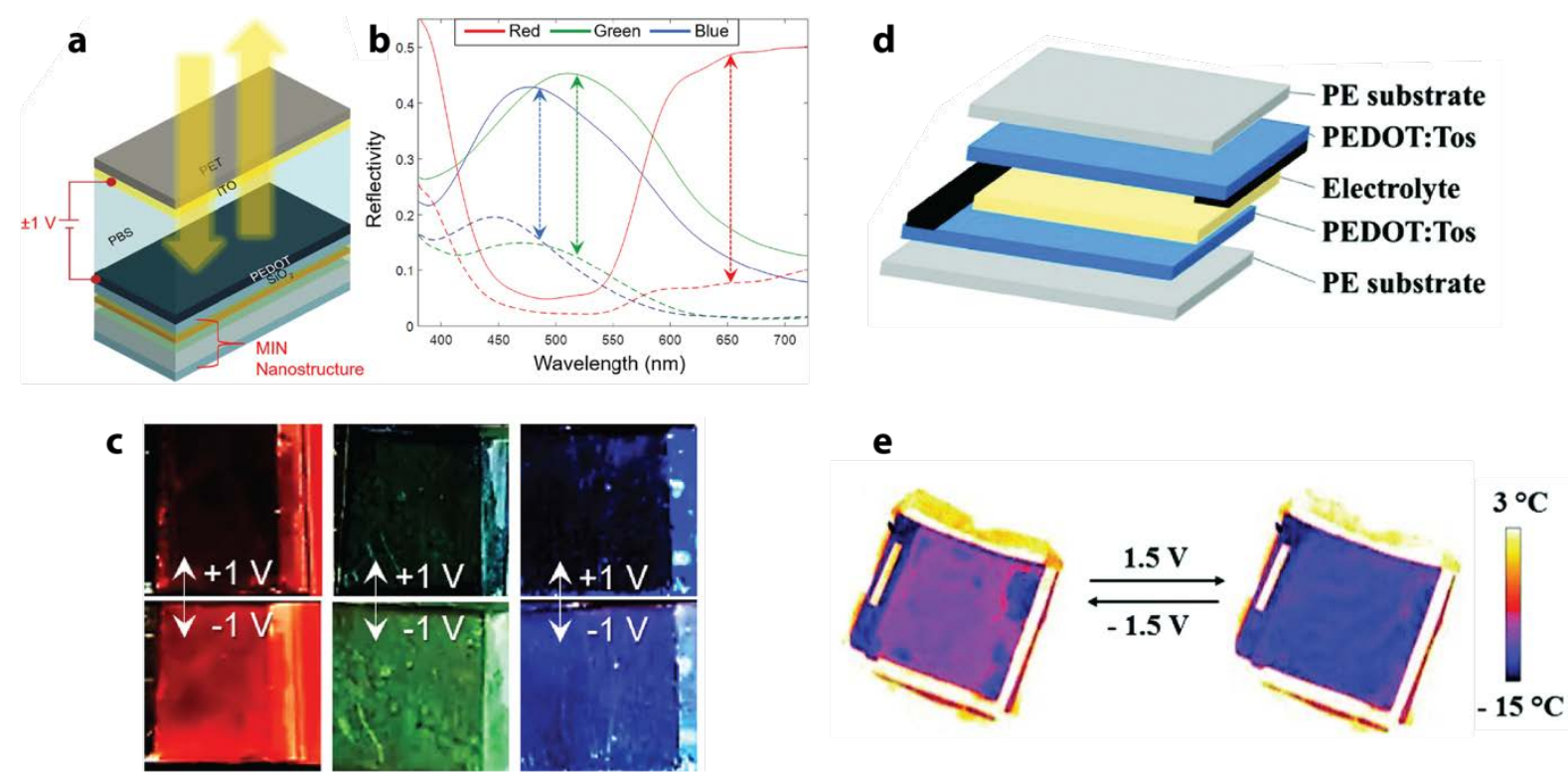

Figure 4. (a-c) Concept of using PEDOT:PSS to control the reflectance from colored high reflectance plasmonic metasurfaces. (a) Schematic of the device, based on a metal-insulatornanohole (MIN) plasmonic metasurface, dielectric protection layers, PEDOT:PSS, PBS electrolyte, and top ITO-coated PET film. For red color, the chromium film was not perforated with nanoholes, which provided better performance. (b) Reflectivity spectra of PEDOTmodified metasurfaces in the on and off states and (d) pictures of around $2 \times 2 \mathrm{~cm}^{2}$-sized hybrid metasurfaces in their off (top) and on (bottom) states. (d-e) PEDOT:Tos devices for infrared electrochromism. (d) Schematic of a vertical electrochromic device, based on polyethylene (PE) as substrates and PEDOT:Tos as electrochromic material. (e) Thermal camera images of a device with the PEDOT facing the camera in its reduced state (left) and oxidized state (right), respectively. The apparent temperature could be dynamically controlled by around $6{ }^{\circ} \mathrm{C}$ for this device. Parts a-c reprinted with permission from the American Chemical Society, ref. [38] copyright 2017; parts $d$ and e reproduced with permission from the Royal Society of Chemistry, ref. [40] copyright 2017. 
a

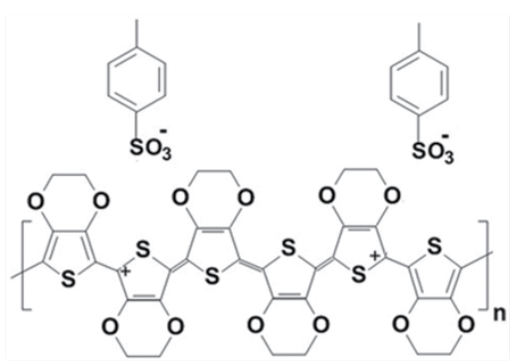

b

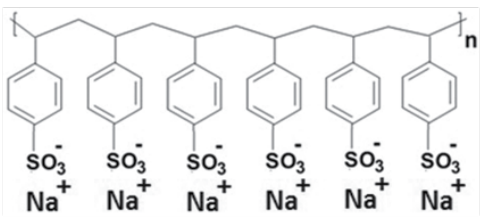

C

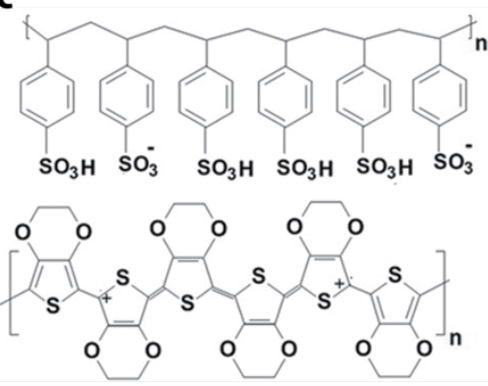

Figure 5. Structure and charge carriers in (a) PEDOT:Tos (majority hole conductor), (b) PSSNa (Cation conductor), and (c) PEDOT:PSS (mixed cation-hole conductor). Reproduced with permission from John Wiley and Sons, ref. [51] copyright 2016. 
a

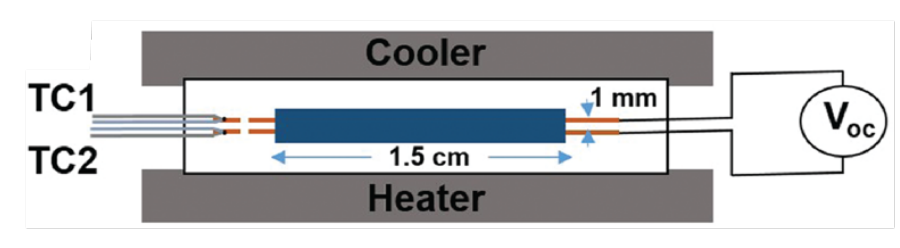

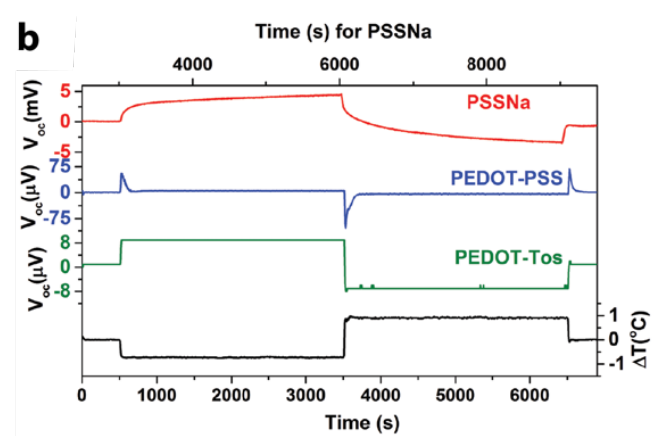

Figure. 6: (a) Schematic of the device for measuring the open circuit voltage $V_{\text {oc }}$ in the temperature gradient. (b) Open circuit voltage vs. time for PEDOT:Tos, PEDOT-PSS, and PSS:Na, at $80 \% \mathrm{RH}$ with $\Delta \mathrm{T}=1{ }^{\circ} \mathrm{C}$. Reproduced with permission from John Wiley and Sons, ref. [51] copyright 2016. 

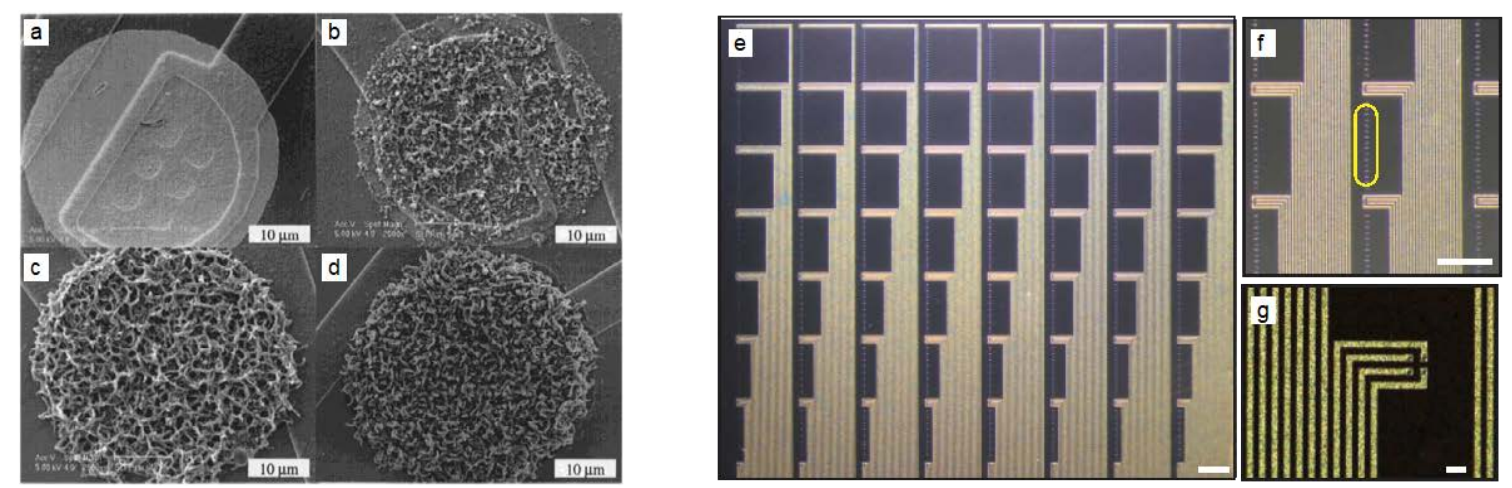

Figure 7. Conducting polymer neural electrodes. (a-d) SEM images of electropolymerized PPy electrodes deposited with (a) $0 \mu \mathrm{C}$, (b) $1 \mu \mathrm{C}$, (c) $4 \mu \mathrm{C}$ and (d) $10 \mu \mathrm{C}$ of charge. The optimal amount of charge depends on the thickness and morphology of the film. In (d) the film has become denser which prevents effective ionic transport. (e) NeuroGrid with 240 PEDOT:PSS electrodes fabricated from chemically polymerized PEDOT:PSS. (scale bar $1 \mathrm{~mm}$ ) (f) Electrodes arranged in $2 \times 2$ configurations with $2 \mathrm{~mm}$ spacing. (scale bar $1 \mathrm{~mm}$ ). (g) The electrodes have a surface area of $10 \mu \mathrm{m}^{2}$ and a $23 \mu \mathrm{m}$ interelectrode spacing. The impedance of the electrodes at $1 \mathrm{kHz}$ is below $100 \mathrm{k} \Omega$ due to the PEDOT:PSS coating. Parts a-d reproduced with permission from John Wiley and Sons, ref. [55] copyright 2001; panels e-g reprinted/adapted from ref. [78] copyright the authors, some rights reserved; exclusive licensee AAAS. Distributed under a Creative Commons Attribution Non-Commercial License 4.0 (CC BY-NC) http://creativecommons.org/licenses/by-nc/4.0/. 

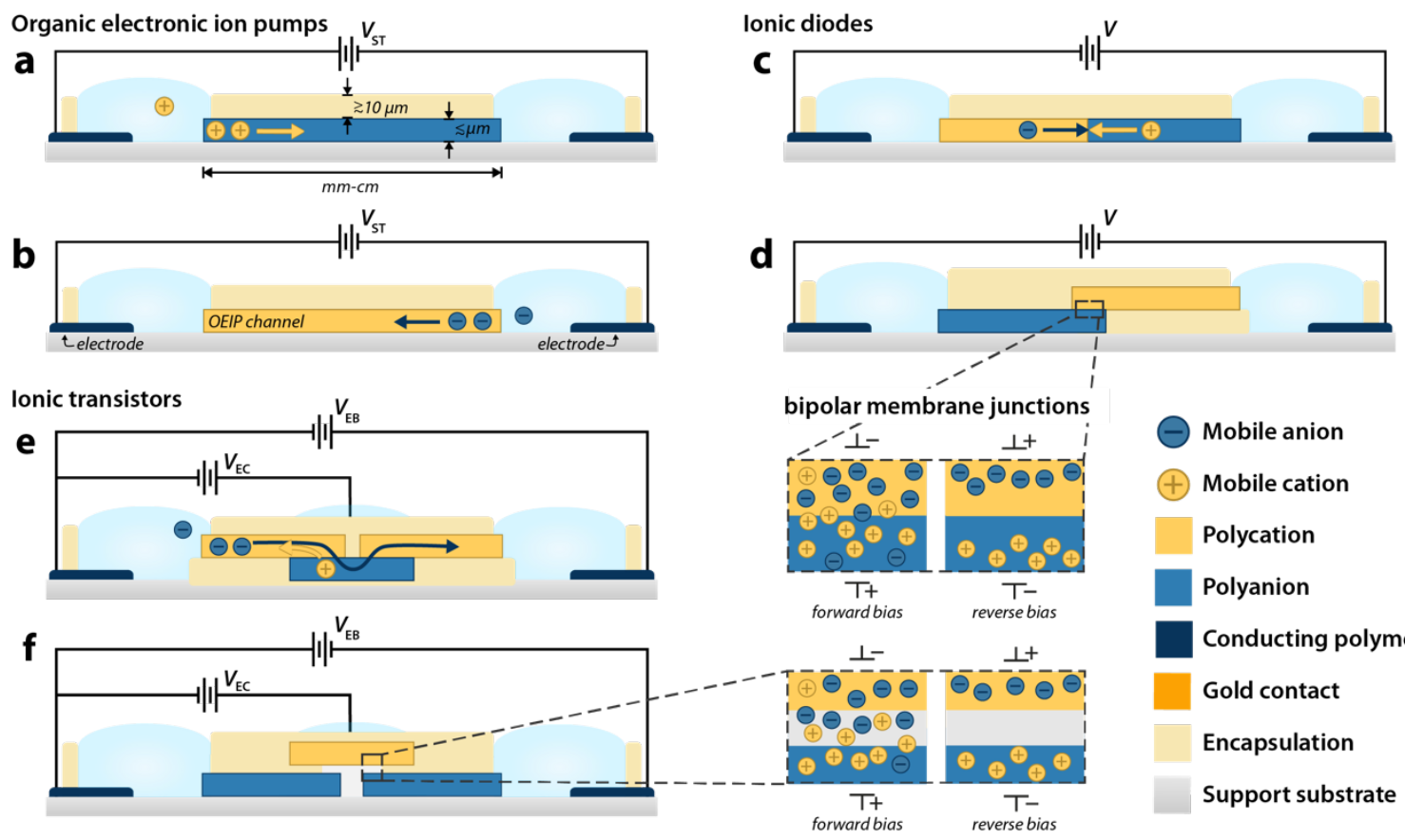

Mobile anion

bipolar membrane junctions

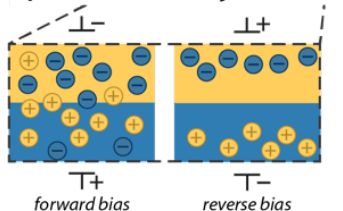

+ Mobile cation

Polycation

Polyanion

Conducting polymer

Gold contact

Encapsulation

Support substrate

Figure 8. Iontronic components and applications. The basic OEIP structure for (a) selective cation transport and (b) selective anion transport. Ionic bipolar membrane diodes with (c) lateral junction and (d) stacked junction; zoom shows accumulation and depletion of the BM junction in forward and reverse bias. (e) npn-IBJT in forward bias. (f) pnp-IBJT with a neutral junction; zoom shows accumulation and depletion of the neutral junction in forward and reverse bias. (g) Figure and caption reproduced with permission from ref. [70]. 

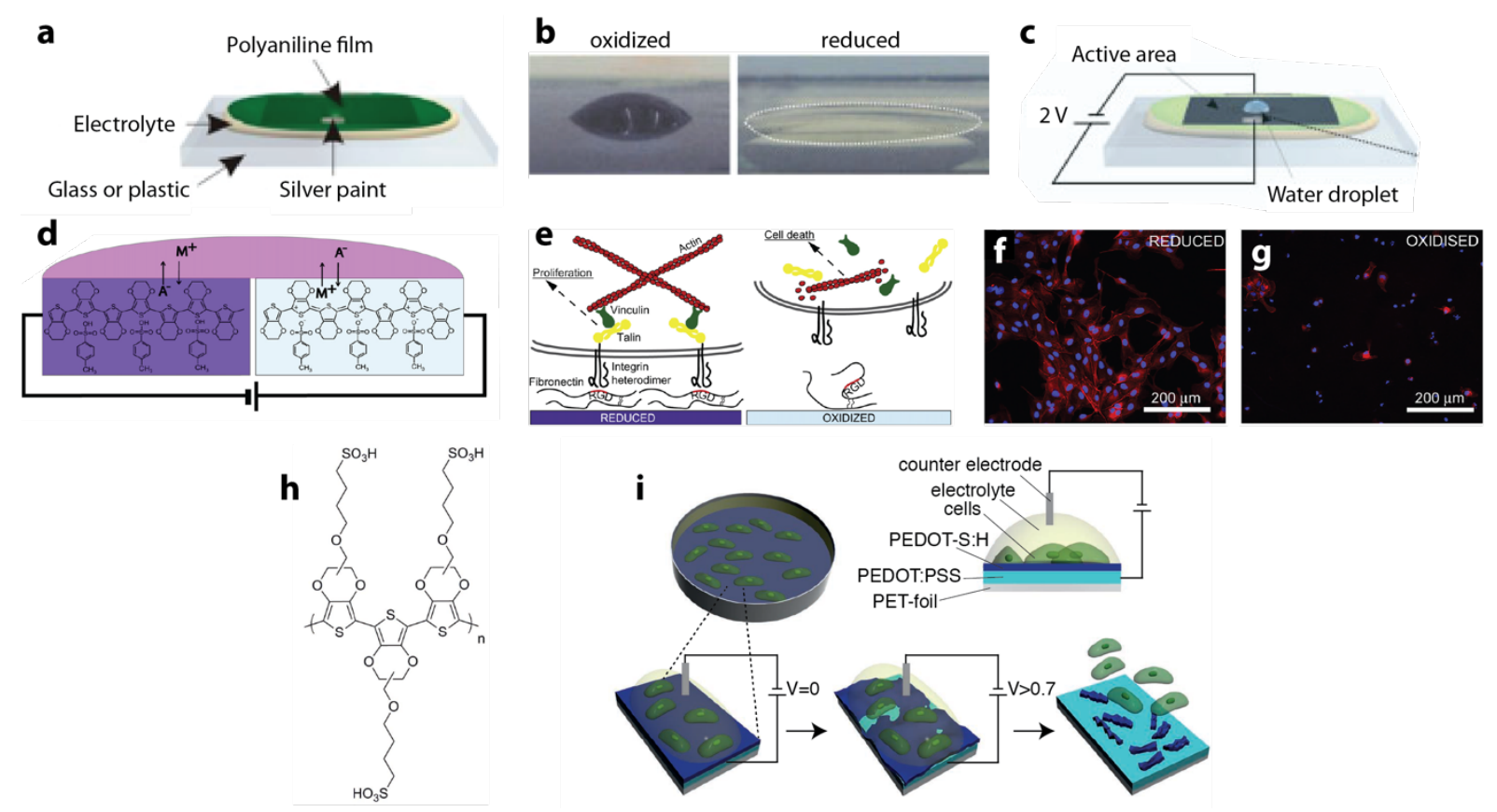

Figure 9. Electronic surface switches to control wettability (a-c), growth (d-g) and detachment (h-i) of epithelial cells. Parts b and c reproduced with permission from John Wiley and Sons, ref. [95] copyright 2004; parts d-g reproduced with permission from Elsevier, ref. [113] copyright 2009; parts h and i reproduced with permission from John Wiley and Sons, ref. [100] copyright 2011. 

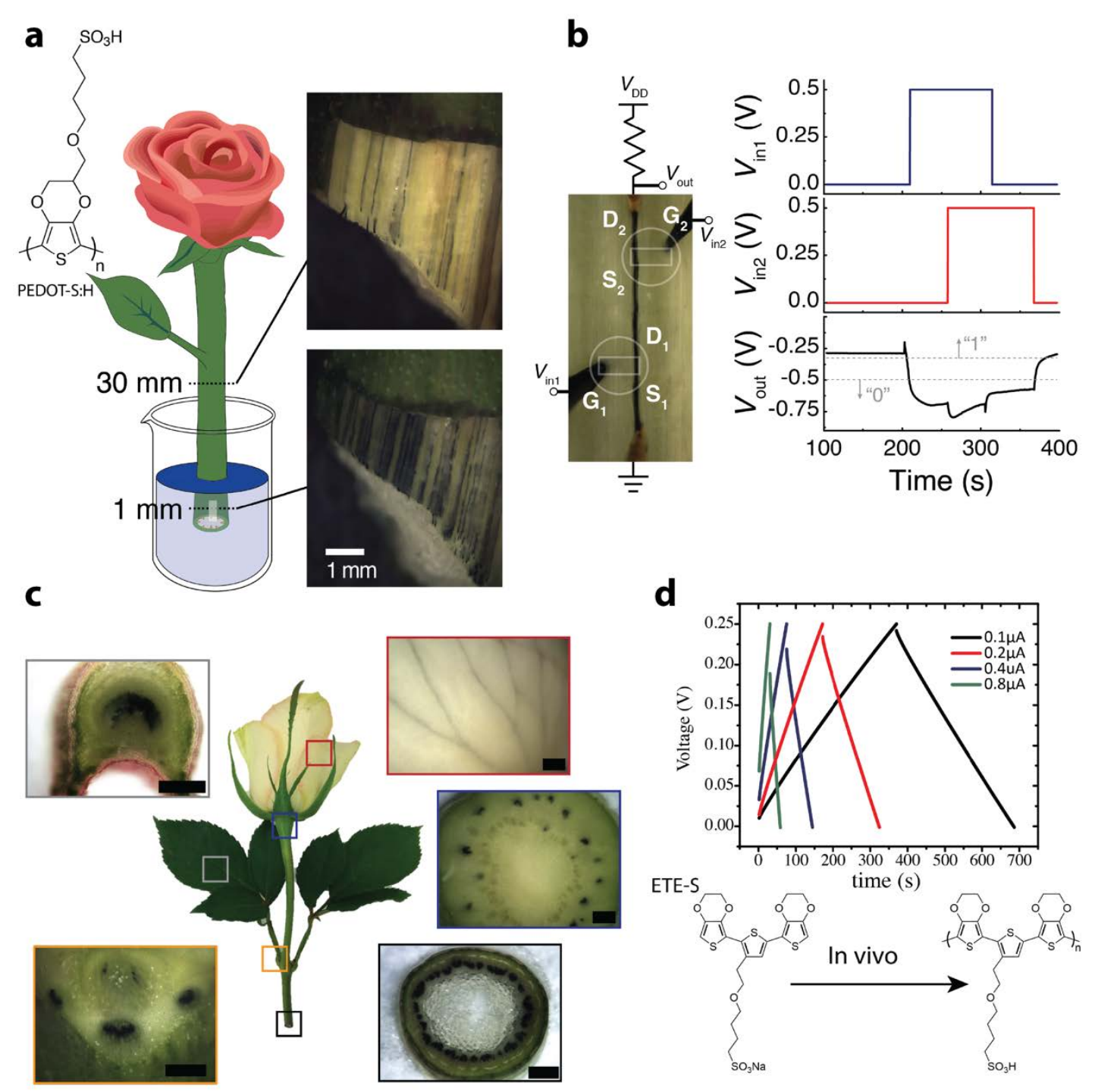

Figure 10. Electronic functionalization of plants. (a) Forming PEDOT-S:H wires in the xylem. A cut rose is immersed in PEDOT-S:H aqueous solution, and PEDOT-S:H is taken up and selforganizes along the xylem forming conducting wires. The optical micrographs show the wires 1 and $30 \mathrm{~mm}$ above the bottom of the stem (bark and phloem were peeled off to reveal the xylem). (b) Logical NOR gate constructed along a single xylem wire. The circuit diagram indicates the location of the two xylem-OECTs and external connections. Voltage traces for $V_{\text {in1 }}, V_{\text {in2 }}$, and $V_{\text {out }}$ illustrate NOR function. The dashed lines on the $V_{\text {out }}$ plot indicate thresholds for defining logical 0 and 1. (c) ETE-S distribution and polymerization in a rose cutting. Rose after immersion in ETE-S aqueous solution for $24 \mathrm{~h}$. Darkened areas in the vascular tissue indicate polymer deposition, scale bar $1 \mathrm{~mm}$. (d) Typical charging-discharging curves for a 
rose supercapacitor. Parts a and b reproduced from ref. [108]; parts c and d reproduced from ref. [109]. 


\section{References}

[1] L. Groenendaal, F. Jonas, D. Freitag, H. Pielartzik, J. R. Reynolds, Adv. Mater. 2000, 12, 481.

[2] Y. Wen, J. Xu, Journal of Polymer Science Part A: Polymer Chemistry 2017, 55, 1121.

[3] K. E. Aasmundtveit, E. J. Samuelsen, L. A. A. Pettersson, O. Inganäs, T. Johansson, R. Feidenhans'l, Synthetic Metals 1999, 101, 561.

[4] A. V. Volkov, K. Wijeratne, E. Mitraka, U. Ail, D. Zhao, K. Tybrandt, J. W. Andreasen, M. Berggren, X. Crispin, I. V. Zozoulenko, Advanced Functional Materials 2017, 1700329.

[5] J. F. Franco-Gonzalez, I. V. Zozoulenko, The Journal of Physical Chemistry B 2017, $121,4299$.

[6] J. F. Franco-Gonzalez, N. Rolland, I. V. Zozoulenko, ACS Applied Materials \& Interfaces 2018.

[7] N. Rolland, J. F. Franco-Gonzalez, R. Volpi, M. Linares, I. V. Zozoulenko, Physical Review Materials 2018, 2, 045605.

[8] M. Modarresi, J. F. Franco-Gonzalez, I. Zozoulenko, Phys Chem Chem Phys 2018, 20, 17188.

[9]

[11]

K. Tybrandt, I. V. Zozoulenko, M. Berggren, Science Advances 2017, 3.

H. S. White, G. P. Kittlesen, M. S. Wrighton, JACS 1984, 106, 5375. Stavrinidou, T. Herve, S. Sanaur, R. M. Owens, G. G. Malliaras, Nature Communications 2013, 4, 2133.

[12] S. G. Bucella, A. Luzio, E. Gann, L. Thomsen, C. R. McNeill, G. Pace, A. Perinot, Z. Chen, A. Facchetti, M. Caironi, Nature Communications 2015, 6, 8394.

[13] A. Laiho, L. Herlogsson, R. Forchheimer, X. Crispin, M. Berggren, Proceedings of the National Academy of Sciences 2011, 108, 15069; A. Giovannitti, D.-T. Sbircea, S. Inal, C. B. Nielsen, E. Bandiello, D. A. Hanifi, M. Sessolo, G. G. Malliaras, I. McCulloch, J. Rivnay, Proceedings of the National Academy of Sciences 2016, 113, 12017.

[14] O. Larsson, A. Laiho, W. Schmickler, M. Berggren, X. Crispin, Adv. Mater. 2011, 23,4764 .

[15] A. Malti, J. Edberg, H. Granberg, Z. U. Khan, J. W. Andreasen, X. Liu, D. Zhao, H. Zhang, Y. Yao, J. W. Brill, I. Engquist, M. Fahlman, L. Wågberg, X. Crispin, M. Berggren, Advanced Science 2016, 3, 1500305.

[16] E. Zeglio, M. Vagin, C. Musumeci, F. N. Ajjan, R. Gabrielsson, X. T. Trinh, N. T. Son, A. Maziz, N. Solin, O. Inganäs, Chem. Mater. 2015, 27, 6385; E. Zeglio, J. Eriksson, R. Gabrielsson, N. Solin, O. Inganäs, Adv. Mater. 2017, 29, 1605787; S. Inal, J. Rivnay, P. Leleux, M. Ferro, M. Ramuz, J. C. Brendel, M. M. Schmidt, M. Thelakkat, G. G. Malliaras, Adv. Mater. 2014, 26, 7450; A. Giovannitti, C. B. Nielsen, D.-T. Sbircea, S. Inal, M. Donahue, M. R. Niazi, D. A. Hanifi, A. Amassian, G. G. Malliaras, J. Rivnay, I. McCulloch, Nature Communications 2016, 7, 13066; H. Sun, M. Vagin, S. Wang, X. Crispin, R. Forchheimer, M. Berggren, S. Fabiano, Adv. Mater. 2018, 30, 1704916.

[17] J. Rivnay, S. Inal, A. Salleo, R. M. Owens, M. Berggren, G. G. Malliaras, Nature Reviews Materials 2018, 3, 17086; E. Zeglio, O. Inganäs, Adv. Mater., 0, 1800941.

[18] D. Nilsson, N. Robinson, M. Berggren, R. Forchheimer, Adv. Mater. 2005, 17, 353. 

14214; E. Mitraka, L. Kergoat, Z. U. Khan, S. Fabiano, O. Douhéret, P. Leclère, M. Nilsson, P. Andersson Ersman, G. Gustafsson, R. Lazzaroni, M. Berggren, X. Crispin, Journal of Materials Chemistry C 2015, 3, 7604.

[20] E. Peter Andersson, W. David, T. Deyu, N. Marie, Å. Jessica, E. Annelie, L. Axel, N. David, S. Mats, N. Petronella, B. Magnus, F. Robert, G. Göran, Flexible and Printed Electronics 2017, 2, 045008.

[21] M. Hamedi, R. Forchheimer, O. Inganas, Nat Mater 2007, 6, 357.

[22] D. T. Simon, E. O. Gabrielsson, K. Tybrandt, M. Berggren, Chem. Rev. 2016, 116, 13009 .

[23] L. H. Jimison, S. A. Tria, D. Khodagholy, M. Gurfinkel, E. Lanzarini, A. Hama, G. G. Malliaras, R. M. Owens, Adv. Mater. 2012, 24, 5919.

[24] P. K. Johansson, D. Jullesson, A. Elfwing, S. I. Liin, C. Musumeci, E. Zeglio, F. Elinder, N. Solin, O. Inganäs, Scientific Reports 2015, 5, 11242.

[25] D. A. Bernards, D. J. Macaya, M. Nikolou, J. A. DeFranco, S. Takamatsu, G. G. Malliaras, J. Mater. Chem. 2008, 18, 116.

[26] C. Yao, Q. Li, J. Guo, F. Yan, I.-M. Hsing, Advanced Healthcare Materials 2015, 4, 528.

[27] A. Campana, T. Cramer, D. T. Simon, M. Berggren, F. Biscarini, Adv. Mater. 2014, 26,3874 .

[28] W. Lee, D. Kim, N. Matsuhisa, M. Nagase, M. Sekino, G. G. Malliaras, T. Yokota, T. Someya, Proceedings of the National Academy of Sciences 2017.

[29] P. Leleux, J. Rivnay, T. Lonjaret, J.-M. Badier, C. Bénar, T. Hervé, P. Chauvel, G. G. Malliaras, Adv. Health. Mater. 2015, 4, 142.

[30] E. J. Fuller, F. E. Gabaly, F. Léonard, S. Agarwal, S. J. Plimpton, R. B. JacobsGedrim, C. D. James, M. J. Marinella, A. A. Talin, Adv. Mater. 2017, 29, 1604310.

[31] Y. Kim, A. Chortos, W. Xu, Y. Liu, J. Y. Oh, D. Son, J. Kang, A. M. Foudeh, C. Zhu, Y. Lee, S. Niu, J. Liu, R. Pfattner, Z. Bao, T.-W. Lee, Science 2018, 360, 998.

[32] Y. van de Burgt, E. Lubberman, E. J. Fuller, S. T. Keene, G. C. Faria, S. Agarwal, M. J. Marinella, A. Alec Talin, A. Salleo, Nat Mater 2017, 16, 414.

[33] P. Gkoupidenis, D. A. Koutsouras, G. G. Malliaras, Nat. Commun. 2017, 8, 15448. [34] S. Fabiano, N. Sani, J. Kawahara, L. Kergoat, J. Nissa, I. Engquist, X. Crispin, M. Berggren, Science Advances 2017, 3, e1700345.

[35] E. Mitraka, M. J. Jafari, M. Vagin, X. Liu, M. Fahlman, T. Ederth, M. Berggren, M. P. Jonsson, X. Crispin, Journal of Materials Chemistry A 2017, 5, 4404.

[36] A. Malti, R. Brooke, X. Liu, D. Zhao, P. Andersson Ersman, M. Fahlman, M. P. Jonsson, M. Berggren, X. Crispin, Journal of Materials Chemistry C 2016, 4, 9680; J. Edberg, D. Iandolo, R. Brooke, X. Liu, C. Musumeci, J. W. Andreasen, D. T. Simon, D. Evans, I. Engquist, M. Berggren, Adv. Funct. Mater. 2016, 26, 6950; R. Brooke, J. Edberg, D. landolo, M. Berggren, X. Crispin, I. Engquist, Journal of Materials Chemistry C 2018, 6, 4663.

[37] K. Xiong, G. Emilsson, A. Maziz, X. Yang, L. Shao, E. W. H. Jager, A. B. Dahlin, Adv. Mat. 2016, 28, 9956.

[38] K. Xiong, D. Tordera, G. Emilsson, O. Olsson, U. Linderhed, M. P. Jonsson, A. B. Dahlin, Nano Lett. 2017, 17, 7033.

[39] L. A. A. Pettersson, T. Johansson, F. Carlsson, H. Arwin, O. Inganäs, Synthetic Metals 1999, 101, 198. 
[40] R. Brooke, E. Mitraka, S. Sardar, M. Sandberg, A. Sawatdee, M. Berggren, X. Crispin, M. P. Jonsson, Journal of Materials Chemistry C 2017, 5, 5824.

[41] G. J. Snyder, E. S. Toberer, Nat. Mater. 2008, 7, 105.

[42] P. Andersson, D. Nilsson, P. O. Svensson, M. Chen, A. Malmström, T. Remonen, T. Kugler, M. Berggren, Adv. Mater. 2002, 14, 1460; Q. Pei, G. Zuccarello, M. Ahlskog, O. Inganäs, Polymer 1994, 35, 1347.

[43] F. Jiao, J. Edberg, D. Zhao, S. Puzinas, Z. U. Khan, P. Mäkie, A. Naderi, T. Lindström, M. Odén, I. Engquist, M. Berggren, X. Crispin, Advanced Sustainable Systems 2018, 2, 1700121.

[44] T. Park, C. Park, B. Kim, H. Shin, E. Kim, Energy \& Environmental Science 2013, 6, 788.

[45] O. Bubnova, Z. U. Khan, A. Malti, S. Braun, M. Fahlman, M. Berggren, X. Crispin, Nat. Mater. 2011, 10, 429.

[46] S. H. Lee, H. Park, S. Kim, W. Son, I. W. Cheong, J. H. Kim, J. Mater. Chem. A 2014, 2,7288 .

[47] G.-H. Kim, L. Shao, K. Zhang, K. P. Pipe, Nat. Mater. 2013, 12, 719.

[48] Q. Wei, M. Mukaida, K. Kirihara, Y. Naitoh, T. Ishida, Applied Physics Express $2014,7$.

[49] H. Wang, U. Ail, R. Gabrielsson, M. Berggren, X. Crispin, Advanced Energy Materials 2015, 5, 1500044.

[50] H. J. V. Tyrrell, D. A. Taylor, C. M. Williams, Nature 1956, 177, 668.

[51] U. Ail, M. J. Jafari, H. Wang, T. Ederth, M. Berggren, X. Crispin, Adv. Funct. Mater. 2016, 26, 6288.

[52] D. R. Merrill, M. Bikson, J. G. R. Jefferys, J. Neurosci. Methods 2005, 141, 171.

[53] A. V. Volkov, K. Wijeratne, E. Mitraka, U. Ail, D. Zhao, K. Tybrandt, J. W. Andreasen, M. Berggren, X. Crispin, I. V. Zozoulenko, Adv. Funct. Mater. 2017, 27.

[54] X. Y. Cui, J. F. Hetke, J. A. Wiler, D. J. Anderson, D. C. Martin, Sens. Actuator APhys. 2001, 93, 8.

[55] X. Y. Cui, V. A. Lee, Y. Raphael, J. A. Wiler, J. F. Hetke, D. J. Anderson, D. C. Martin, J. Biomed. Mater. Res. 2001, 56, 261.

[56] M. Asplund, T. Nyberg, O. Inganas, Polym. Chem. 2010, 1, 1374.

[57] C. Weidlich, K. M. Mangold, K. Juttner, Electrochim. Acta 2005, 50, 1547; J. B. Xia, N. Masaki, K. J. Jiang, S. Yanagida, J. Mater. Chem. 2007, 17, 2845.

[58] M. Asplund, H. von Holst, O. Inganas, Biointerphases 2008, 3, 83.

[59] G. C. Li, P. G. Pickup, Phys. Chem. Chem. Phys. 2000, 2, 1255; J. Bobacka, A. Lewenstam, A. Ivaska, J. Electroanal. Chem. 2000, 489, 17.

[60] D. H. Kim, M. Abidian, D. C. Martin, J. Biomed. Mater. Res. Part A 2004, 71A, 577; T. Nyberg, O. Inganas, H. Jerregard, Biomed. Microdevices 2002, 4, 43.

[61] X. Y. Cui, D. C. Martin, Sens. Actuators, B 2003, 89, 92.

[62] Y. H. Xiao, D. C. Martin, X. Y. Cui, M. Shenai, Applied Biochemistry and Biotechnology 2006, 128, 117; Y. H. Xiao, X. Y. Cui, J. M. Hancock, M. B. Bouguettaya, J. R. Reynolds, D. C. Martin, Sens. Actuators, B 2004, 99, 437.

[63] X. D. Wang, X. S. Gu, C. W. Yuan, S. J. Chen, P. Y. Zhang, T. Y. Zhang, J. Yao, F. Chen, G. Chen, J. Biomed. Mater. Res. Part A 2004, 68A, 411; P. M. George, A. W. Lyckman, D. A. LaVan, A. Hegde, Y. Leung, R. Avasare, C. Testa, P. M. Alexander, R. Langer, M. Sur, Biomaterials 2005, 26, 3511. 

Inganas, H. von Holst, Biomed. Mater. 2009, 4.

[65] E. M. Thaning, M. L. M. Asplund, T. A. Nyberg, O. W. Inganas, H. von Hoist, J. Biomed. Mater. Res. Part B 2010, 93B, 407.

[66] R. A. Green, C. M. Williams, N. H. Lovell, L. A. Poole-Warren, J. Mater. Sci.: Mater. Med. 2008, 19, 1625.

[67] E. Castagnola, L. Maiolo, E. Maggiolini, A. Minotti, M. Marrani, F. Maita, A. Pecora, G. N. Angotzi, A. Ansaldo, M. Boffini, L. Fadiga, G. Fortunato, D. Ricci, IEEE Transactions on Neural Systems and Rehabilitation Engineering 2015, 23, 342; L. K. Christi, C. Kasey, R. Erika, N. A. Ingrid, B. Dennis, D. Zhanhong, D. Y. K. Takashi, L. Xiliang, J. W. Douglas, X. T. Cui, J. Neural. Eng. 2015, 12, 016008; S. Chen, W. Pei, Q. Gui, R. Tang, Y. Chen, S. Zhao, H. Wang, H. Chen, Sensors and Actuators A: Physical 2013, 193, 141.

[68] C. Boehler, F. Oberueber, S. Schlabach, T. Stieglitz, M. Asplund, ACS Applied Materials \& Interfaces 2017, 9, 189.

[69] L. Ouyang, B. Wei, C.-c. Kuo, S. Pathak, B. Farrell, D. C. Martin, Sci. Adv. 2017, 3. [70] D. Khodagholy, T. Doublet, M. Gurfinkel, P. Quilichini, E. Ismailova, P. Leleux, T. Herve, S. Sanaur, C. Bernard, G. G. Malliaras, Adv. Mater. 2011, 23, H268.

[71] D. Khodagholy, T. Doublet, P. Quilichini, M. Gurfinkel, P. Leleux, A. Ghestem, E. Ismailova, T. Herve, S. Sanaur, C. Bernard, G. G. Malliaras, Nat. Commun. 2013, 4.

[72] R. S. Fisher, A. L. Velasco, Nature Reviews Neurology 2014, 10, 261; R. L. Testerman, M. T. Rise, P. H. Stypulkowski, IEEE Engineering in Medicine and Biology Magazine 2006, 25, 74.

[73] N. K. Guimard, N. Gomez, C. E. Schmidt, Prog. Polym. Sci. 2007, 32, 876.

[74] M. R. Abidian, K. A. Ludwig, T. C. Marzullo, D. C. Martin, D. R. Kipke, Adv. Mater. 2009, 21, 3764; B. Gytis, M. Emma, C. Elisa, A. Alberto, M. Alberto, A. Gian Nicola, V. Alessandro, R. Davide, P. Stefano, F. Luciano, J. Neural. Eng. 2011, 8, 066013.

[75] M. R. Abidian, D.-H. Kim, D. C. Martin, Adv. Mater. 2006, 18, 405.

[76] S. M. Richardson-Burns, J. L. Hendricks, D. C. Martin, J. Neural. Eng. 2007, 4, L6.

[77] D. Khodagholy, J. N. Gelinas, T. Thesen, W. Doyle, O. Devinsky, G. G. Malliaras, G. Buzsáki, Nat. Neurosci. 2014, 18, 310.

[78] D. Khodagholy, J. N. Gelinas, Z. F. Zhao, M. Yeh, M. Long, J. D. Greenlee, W. Doyle, O. Devinsky, G. Buzsaki, Sci. Adv. 2016, 2.

[79] Y. Wang, C. X. Zhu, R. Pfattner, H. P. Yan, L. H. Jin, S. C. Chen, F. Molina-Lopez, F. Lissel, J. Liu, N. I. Rabiah, Z. Chen, J. W. Chung, C. Linder, M. F. Toney, B. Murmann, Z. Bao, Sci. Adv. 2017, 3; I. R. Minev, P. Musienko, A. Hirsch, Q. Barraud, N. Wenger, E. M. Moraud, J. Gandar, M. Capogrosso, T. Milekovic, L. Asboth, R. F. Torres, N. Vachicouras, Q. Liu, N. Pavlova, S. Duis, A. Larmagnac, J. Vörös, S. Micera, Z. Suo, G. Courtine, S. P. Lacour, Science 2015, 347, 159; K. Tybrandt, D. Khodagholy, B. Dielacher, F. Stauffer, F. Renz Aline, G. Buzsáki, J. Vörös, Adv. Mater. 2018, 30, 1706520.

[80] D. Svirskis, J. Travas-Sejdic, A. Rodgers, S. Garg, J. Controlled Release 2010, 146, 6.

[81] G. Tarabella, F. M. Mohammadi, N. Coppedè, F. Barbero, S. lannotta, C. Santato, F. Cicoira, Chemical Science 2013, 4, 1395; D. Khodagholy, J. N. Gelinas, T. Thesen, W. Doyle, O. Devinsky, G. G. Malliaras, G. Buzsáki, Nature Neuroscience 2015, 18, 310; J. Rivnay, S. Inal, A. Salleo, R. M. Owens, M. Berggren, G. G. Malliaras, Nature Reviews Materials 2018, 3, 17086; T. Arbring Sjöström, M. Berggren, E. O. Gabrielsson, P. Janson, D. J. Poxson, M. Seitanidou, D. T. Simon, Advanced Materials Technologies 2018, 3, 1700360. 
[82] K. Kontturi, L. Murtomäki, J. A. Manzanares, lonic Transport Processes: In Electrochemistry and Membrane Science, 2008.

[83] E. O. Gabrielsson, K. Tybrandt, M. Berggren, Lab on a Chip 2012, 12, 2507.

[84] E. O. Gabrielsson, M. Berggren, K. Tybrandt, M. Berggren, Biomicrofluidics 2013, 7, 64117.

[85] J. Isaksson, P. Kjäll, D. Nilsson, N. Robinson, M. Berggren, A. Richter-Dahlfors, Nat. Mater. 2007, 6, 673; K. Tybrandt, K. C. Larsson, S. Kurup, D. T. Simon, P. Kjall, J. Isaksson, M. Sandberg, E. W. H. Jager, A. Richter-Dahlfors, M. Berggren, Advanced Materials 2009, 21, 4442.

[86] D. T. Simon, S. Kurup, K. C. Larsson, R. Hori, K. Tybrandt, M. Goiny, E. W. H. Jager, M. Berggren, B. Canlon, A. Richter-Dahlfors, Nature Materials 2009, 8, 742.

[87] A. Jonsson, S. Inal, I. Uguz, A. J. Williamson, L. Kergoat, J. Rivnay, D. Khodagholy, M. Berggren, C. Bernard, G. G. Malliaras, D. T. Simon, Proc. Natl. Acad. Sci. 2016, 113, 9440; A. Williamson, J. Rivnay, L. Kergoat, A. Jonsson, S. Inal, I. Uguz, M. Ferro, A. Ivanov, T. A. Sjöström, D. T. Simon, M. Berggren, G. G. Malliaras, C. Bernard, Adv. Mater. 2015, 27, 3138.

[88] A. Jonsson, Z. Song, D. Nilsson, B. A. Meyerson, D. T. Simon, B. Linderoth, M. Berggren, Sci. Adv. 2015, 1, e1500039.

[89] D. J. Poxson, M. Karady, R. Gabrielsson, A. Y. Alkattan, A. Gustavsson, S. M. Doyle, S. Robert, K. Ljung, M. Grebe, D. T. Simon, M. Berggren, Proc. Natl. Acad. Sci. 2017, 114, 4597. [90] K. Tybrandt, K. C. Larsson, A. Richter-Dahlfors, M. Berggren, Proc. Natl. Acad. Sci. 2010, 107, 9929; K. Tybrandt, E. O. Gabrielsson, M. Berggren, Journal of the American Chemical Society 2011, 133, 10141.

[91] E. O. Gabrielsson, K. Tybrandt, M. Berggren, Biomicrofluidics 2014, 8, 64116; K. Tybrandt, R. Forchheimer, M. Berggren, Nat. Commun. 2012, 3, 871.

[92] E. O. Gabrielsson, P. Janson, K. Tybrandt, D. T. Simon, M. Berggren, Adv. Mater. 2014, 26, 5143.

[93] A. Jonsson, T. Arbring Sjöström, K. Tybrandt, M. Berggren, D. T. Simon, Sci. Adv. 2016, 2, e1601340.

[94] C. M. Proctor, A. Slézia, A. Kaszas, A. Ghestem, I. del Agua, A.-M. Pappa, C. Bernard, A. Williamson, G. G. Malliaras, Sci. Adv. 2018, 4, eaau1291; I. Uguz, C. M. Proctor, V. F. Curto, A.-M. Pappa, M. J. Donahue, M. Ferro, R. M. Owens, D. Khodagholy, S. Inal, G. G. Malliaras, Adv. Mater. 2017, 1701217, 1701217.

[95] J. Isaksson, C. Tengstedt, M. Fahlman, N. Robinson, M. Berggren, Adv. Mater. 2004, 16, 316.

[96] L. Robinson, J. Isaksson, N. Robinson, M. Berggren, Surface Science 2006, 600, L148.

[97] J. Y. Wong, R. Langer, D. E. Ingber, Proceedings of the National Academy of Sciences 1994, 91, 3201.

[98] M. H. Bolin, K. Svennersten, D. Nilsson, A. Sawatdee, E. W. H. Jager, A. RichterDahlfors, M. Berggren, Adv. Mater. 2009, 21, 4379.

[99] C. Waymouth, In Vitro 1974, 10, 97.

[100] K. M. Persson, R. Karlsson, K. Svennersten, S. Löffler, E. W. H. Jager, A. RichterDahlfors, P. Konradsson, M. Berggren, Adv. Mater. 2011, 23, 4403.

[101] M. Hamedi, A. Elfwing, R. Gabrielsson, O. Ingan s, Small 2012, 9, 363.

[102] A. Elfwing, F. G. Bäcklund, C. Musumeci, O. Inganäs, N. Solin, Journal of Materials Chemistry C 2015, 3, 6499. 
[103] P. K. Johansson, D. Jullesson, A. Elfwing, S. I. Liin, C. Musumeci, E. Zeglio, F. Elinder, N. Solin, O. Ingan s, Scientific Reports 2015, 5, 581.

[104] F. G. Bäcklund, A. Elfwing, C. Musumeci, F. Ajjan, V. Babenko, W. Dzwolak, N. Solin, O. Inganäs, Soft Matter 2017, 13, 4412.

[105] U. S. Herrmann, A. K. Schütz, H. Shirani, D. Huang, D. Saban, M. Nuvolone, B. Li, B. Ballmer, A. K. O. Åslund, J. J. Mason, E. Rushing, H. Budka, S. Nyström, P. Hammarström, A. Böckmann, A. Caflisch, B. H. Meier, K. P. R. Nilsson, S. Hornemann, A. Aguzzi, Science Translational Medicine 2015, 7, 299 ra123.

[106] H. Shirani, M. Linares, C. J. Sigurdson, M. Lindgren, P. Norman, K. P. R. Nilsson, Chemistry - A European Journal 2015, 21, 15133.

[107] A. Elfwing, C. S. Ponseca, L. Ouyang, A. Urbanowicz, A.-n. Krotkus, D. Tu, R. Forchheimer, O. Inganäs, Adv. Funct. Mater. 2018, 28, 1706595.

[108] E. Stavrinidou, R. Gabrielsson, E. F. Gomez, X. Crispin, O. Nilsson, D. T. Simon, M. Berggren, Sci. Adv. 2015, 1, e1501136.

[109] E. Stavrinidou, R. Gabrielsson, K. P. R. Nilsson, S. K. Singh, J. F. Franco-Gonzalez, A. V. Volkov, M. P. Jonsson, A. Grimoldi, M. Elgland, I. V. Zozoulenko, D. T. Simon, M. Berggren, Proc. Natl. Acad. Sci. 2017, 114, 2807.

[110] J. F. Franco-Gonzalez, E. Pavlopoulou, E. Stavrinidou, R. Gabrielsson, D. T. Simon, M. Berggren, I. V. Zozoulenko, Nanoscale 2017, 9, 13717.

[111] W. Lee, D. Kim, N. Matsuhisa, M. Nagase, M. Sekino, G. G. Malliaras, T. Yokota, T. Someya, Proc Natl Acad Sci U S A 2017, 114, 10554.

[112] Y. Kim, A. Chortos, W. Xu, Y. Liu, J. Y. Oh, D. Son, J. Kang, A. M. Foudeh, C. Zhu, Y. Lee, S. Niu, J. Liu, R. Pfattner, Z. Bao, T. W. Lee, Science 2018, 360, 998.

[113] K. Svennersten, M. H. Bolin, E. W. H. Jager, M. Berggren, A. Richter-Dahlfors, Biomaterials 2009, 30, 6257. 